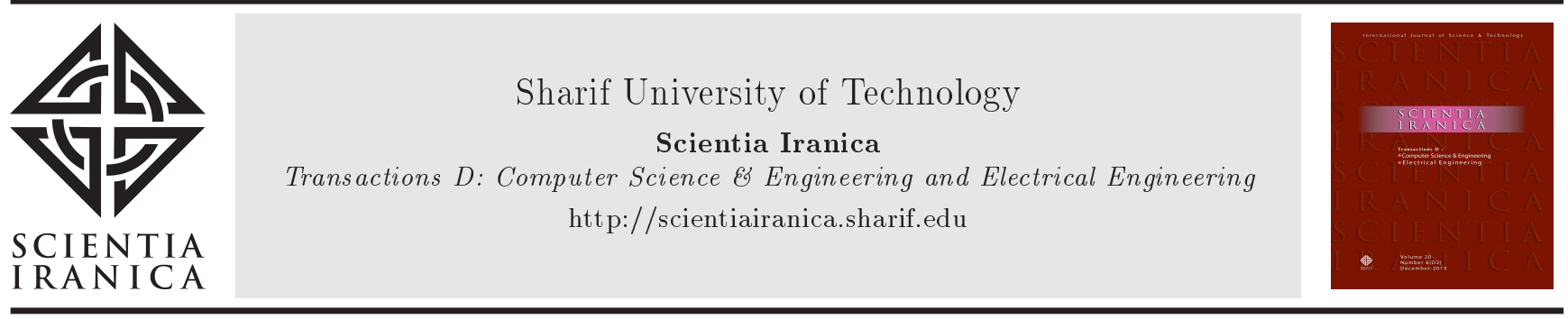

\title{
Two low computational complexity improved multiband-structured subband adaptive filter algorithms
}

\author{
M. Shams Esfand Abadi ${ }^{a, *}$, J.H. Husøy ${ }^{b}$, and M.J. Ahmadi ${ }^{a}$ \\ a. Faculty of Electrical Engineering, Shahid Rajaee Teacher Training University, Tehran, P.O. Box: 16785-163, Iran. \\ b. Department of Electrical Engineering and Computer Science, Faculty of Science and Engineering, University of Stavanger, \\ Norway.
}

Received 10 July 2018; received in revised form 25 May 2019; accepted 10 August 2019

\author{
KEYWORDS \\ Improved Multiband- \\ structured Subband \\ Adaptive Filter \\ (IMSAF); \\ Selective Partial \\ Update (SPU); \\ Set-Membership \\ $(\mathrm{SM})$ \\ Convergence rate; \\ Computational \\ complexity.
}

\begin{abstract}
The Improved Multiband-structured Subband Adaptive Filter (IMSAF) applies the input regressors at each subband to speed up the convergence rate of MultibandStructure Subband Adaptive Filter (MSAF). When the projection order increases, the convergence rate of the IMSAF algorithm improves at the cost of increased complexity. The present research introduces two new IMSAF algorithms with low computational complexity feature. In the first algorithm, the Selective Partial Update (SPU) approach is extended to IMSAF algorithms and SPU-IMSAF is established. In SPU-IMSAF, the filter coefficients are partially updated at each subband for every adaptation. In the second algorithm, the Set-Membership (SM) strategy is utilized in IMSAF and SM-IMSAF is established. The SM-IMSAF has a fast convergence rate, low steady-state error, and low computational complexity features at the same time. Also, by combining SM and SPU methods, the SM-SPU-IMSAF is introduced. Simulation results demonstrate the good performance of the proposed algorithms.
\end{abstract}

(C) 2021 Sharif University of Technology. All rights reserved.

\section{Introduction}

Adaptive filters are utilized in many applications such as system identification, system inversion, signal prediction, and multisensor interference cancellation [13]. In these applications, the generated signals are processed to identify the characteristics of the unknown system. This aim is successfully achieved using adaptive filters. The adaptive filters apply a recursive

*. Corresponding author. Tel./Fax: +982122970003 E-mail addresses: mshams@sru.ac.ir (M. Shams Esfand Abadi); john.h.husoy@uis.no (J.H.Husøy)

doi: $10.24200 /$ sci.2019.51327.2116 algorithm to design itself. The algorithm updates the weight coefficients through successive iterations and, finally, converges to the optimal Wiener-Hopf solution when signals are statistically stationary. The performance of an adaptive filtering algorithm is evaluated by the rate of convergence, misadjustment, and computational complexity features. The conventional Least Mean Squares (LMS) adaptive filter algorithm has the advantage of being very simple and easy to implement with very low computational complexity. However, when the input signal is highly colored, the LMS convergence slows down $[3,4]$.

To improve the convergence behavior of LMS, various adaptive algorithms such as the Affine Projection Algorithm (APA) and Multiband-Structured 
Subband Adaptive Filter (MSAF) were proposed [510]. The APA is one of the important families of adaptive filter algorithm. Since the interplay between the computational complexity and the performance of adaptive signal processing systems is important [5], several types of APAs have been proposed. For example, in Selective Partial Update APA (SPU-APA), the filter coefficients are partially updated at each time iteration [11-15]. This algorithm has close performance to conventional APA. Also, the SPU-MSAF was proposed in $[16,17]$. In SPU-MSAF algorithm, the filter coefficients were partially updated rather than the entire filter at every adaptation. Recently, the Sign-Regressor MSAF (SRMSAF) and sign-error MSAF were introduced in $[18,19]$. In [18], the sign of input regressors was applied in update equation. The SR-MSAF was successfully extended to adaptive distributed network in [20].

There is another class of adaptive filter algorithms featuring high convergence speed, low computational complexity, and low steady state error at the same time. These algorithms are established based on SetMembership (SM) approach [21]. The SM Normalized LMS (SM-NLMS) was introduced in [22]. The SMAPA and SM-MSAF were derived in $[16,23]$, respectively.

To increase the convergence speed of MSAF, the Improved Multiband-Structured Subband Adaptive Filter (IMSAF) was developed [24-26]. This algorithm utilizes multiple input regressors in each subband during the adaptation. Therefore, the computational complexity of IMSAF increases. To reduce the computational complexity of IMSAF, two approaches were introduced in [27]. In the proposed algorithms, the input regressors were optimally selected at each subband during the adaptation. In Selective Regressor IMSAF (SR-IMSAF), this selection was fixed and in Dynamic SR-IMSAF (DSRIMSAF), this selection was dynamic. This paper proposes two new solutions to reduce the computational load of the IMSAF algorithm. In the first approach, the SPU method is extended to IMSAF algorithm. In SPU-IMSAF, the filter coefficients are partially updated at each subband for every adaptation. The SPU-IMSAF has close performance to IMSAF. To have fast convergence speed, low steady-state error, and low computational complexity at the same time, the SM method is utilized in IMSAF and SM-IMSAF is proposed. Finally, by combination of SM and SPU approaches, the SM-SPUIMSAF is established.

This paper is organized as follows. In Section 2, NLMS, SPU-NLMS, and SM-NLMS algorithms are reviewed. Section 3 reviews the MSAF and IMSAF algorithms. Section 4 introduces the SPU-IMSAF algorithm. The SMIMSAF and SM-SPU-IMSAF algorithms are presented in Section 5. The computational complexity of the proposed algorithms is discussed in Section 6. Finally, before concluding the paper, we demonstrate the usefulness of the introduced algorithms by presenting several experimental results.

Throughout the paper, the following notations are used:

|.| Norm of a scalar

$\|\cdot\|^{2} \quad$ Squared Euclidean norm of a vector

$\operatorname{Tr}($.$) \quad Trace of a matrix$

$(.)^{T} \quad$ Transpose of a vector or a matrix

$E\{\cdot\} \quad$ Expectation operator

\section{Background on NLMS, SPU-NLMS, and SM-NLMS algorithms}

Consider a linear data model for $d(n)$ :

$$
d(n)=\mathbf{x}^{T}(n) \mathbf{w}^{o}+v(n)
$$

where $\mathbf{w}^{o}$ is an unknown $M$-dimensional vector that we aim to estimate, $v(n)$ is the measurement noise with variance $\sigma_{v}^{2}$, and $\mathbf{x}(n)=[x(n), x(n-1), \cdots, x(n-$ $M+1)]^{T}$ denotes an $M$-dimensional input (regressor) vector. It is assumed that $v(n)$ is zero mean, white, Gaussian, and independent of $\mathbf{x}(n)$. It is well known that the NLMS algorithm can be derived from the solution of the following optimization problem:

$$
\min \|\mathbf{w}(n+1)-\mathbf{w}(n)\|^{2},
$$

subject to:

$$
d(n)=\mathbf{x}^{T}(n) \mathbf{w}(n+1),
$$

where $\mathbf{w}(n)=\left[w_{0}(n), w_{1}(n), \cdots, w_{M-1}(n)\right]^{T}$ is the vector of adaptive filter coefficients. Using the method of Lagrange multipliers to solve this optimization problem, the following update equation for NLMS algorithm is given by:

$$
\mathbf{w}(n+1)=\mathbf{w}(n)+\mu \frac{\mathbf{x}(n)}{\|\mathbf{x}(n)\|^{2}} e(n),
$$

where $e(n)=d(n)-\mathbf{x}^{T}(n) \mathbf{w}(n)$ and $\mu$ is the step-size.

Now, partition the input signal vector and the vector of filter coefficients into $B$ blocks, each of length $L(B=M / L$ and is an integer), which are defined as follows:

$$
\begin{aligned}
& \mathbf{x}(n)=\left[\mathbf{x}_{1}^{T}(n), \mathbf{x}_{2}^{T}(n), \cdots, \mathbf{x}_{B}^{T}(n)\right]^{T}, \\
& \mathbf{w}(n)=\left[\mathbf{w}_{1}^{T}(n), \mathbf{w}_{2}^{T}(n), \cdots, \mathbf{w}_{B}^{T}(n)\right]^{T} .
\end{aligned}
$$

The SPU-NLMS algorithm for a single block update at every iteration minimizes the following optimization problem:

$$
\min \left\|\mathbf{w}_{j}(n+1)-\mathbf{w}_{j}(n)\right\|^{2},
$$


subject to Eq. (3), where $j$ denotes the index of the block that should be updated. Again, by using the method of Lagrange multipliers, the update equation for SPU-NLMS is established as follows:

$$
\mathbf{w}_{j}(n+1)=\mathbf{w}_{j}(n)+\mu \frac{\mathbf{x}_{j}(n)}{\left\|\mathbf{x}_{j}(n)\right\|^{2}} e(n),
$$

where $j=\arg \max \left\|\mathbf{x}_{i}(n)\right\|^{2}$ for $1 \leq i \leq B$ [11].

The SM-NLMS algorithm minimizes Eq. (2) subject to $\mathbf{w}(n+1) \in \Psi(n)$, where:

$$
\Psi(n)=\left\{\mathbf{w} \in \mathcal{R}^{M}:\left|d(n)-\mathbf{x}^{T}(n) \mathbf{w}\right| \leq \gamma\right\} .
$$

The set $\Psi(n)$ is referred to as the constraint set, and its boundaries are hyperplanes. Also, $\gamma$ is the magnitude of the error bound.

This aim is achieved by an orthogonal projection of the previous estimate of $\mathbf{w}$ onto the closest boundary of $\Psi(n)$. Through this, the recursion for the SM-NLMS is obtained by [22]:

$$
\mathbf{w}(n+1)=\mathbf{w}(n)+\alpha(n) \frac{\mathbf{x}(n)}{\|\mathbf{x}(n)\|^{2}} e(n),
$$

where:

$$
\alpha(n)= \begin{cases}1-\frac{\gamma}{|e(n)|} & \text { if }|e(n)|>\gamma \\ 0 & \text { otherwise }\end{cases}
$$

\section{Background on MSAF and IMSAF algorithms}

Figure 1 shows the structure of the MSAF [7]. In this figure, $\mathbf{f}_{0}, \mathbf{f}_{1}, \cdots, \mathbf{f}_{N-1}$ and $\mathbf{g}_{0}, \mathbf{g}_{1}, \cdots, \mathbf{g}_{N-1}$, are analysis and synthesis filter unit pulse responses of an $N$ channel orthogonal perfect reconstruction critically sampled filter bank system. $x_{i}(n)$ and $d_{i}(n)$ are nondecimated subband signals. It is important to note that $n$ represents the index of the original sequences and $k$ denotes the index of the decimated sequences $(k=\operatorname{floor}(n / N))$. The decimated output signal is defined as:

$$
y_{i, D}(k)=\mathbf{x}_{i}^{T}(k) \mathbf{w}(k),
$$

where:

$$
\mathbf{x}_{i}(k)=\left[x_{i}(k N), x_{i}(k N-1), \cdots, x_{i}(k N-M+1)\right]^{T},
$$

and:

$$
\mathbf{w}(k)=\left[w_{0}(k), w_{1}(k), \cdots, w_{M-1}(k)\right]^{T} .
$$

Also, the decimated subband error signal is expressed as $e_{i, D}(k)=d_{i, D}(k)-\mathbf{x}_{i}^{T}(k) \mathbf{w}(k)$. The filter update equation for MSAF can be established through the following cost function:

$$
\min \|\mathbf{w}(k+1)-\mathbf{w}(k)\|^{2},
$$

subject to $d_{i, D}(k)=\mathbf{x}_{i}^{T}(k) \mathbf{w}(k+1)$. Using Lagrangemultipliers approach to solving this optimization problem leads to the filter coefficients update equation for MSAF as follows:

$$
\mathbf{w}(k+1)=\mathbf{w}(k)+\mu \sum_{i=0}^{N-1} \frac{\mathbf{x}_{i}(k)}{\left\|\mathbf{x}_{i}(k)\right\|^{2}} e_{i, D}(k) .
$$

The IMSAF minimizes Eq. (12), subject to $\mathbf{d}_{i, D}(k)=$ $\mathbf{X}_{i}^{T}(k) \mathbf{w}(k+1)$, where:

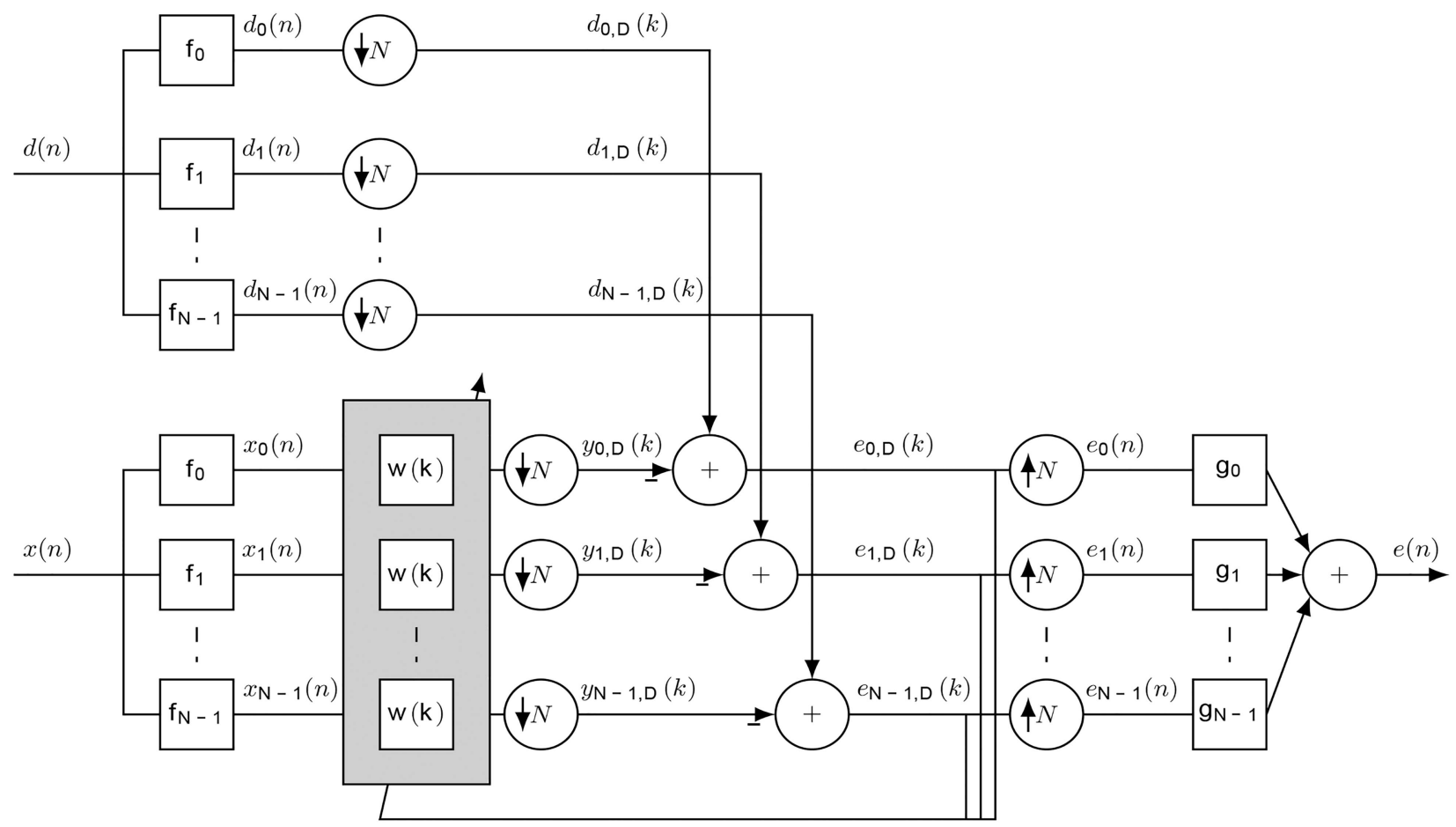

Figure 1. Structure of the Multiband-Structure Subband Adaptive Filter (MSAF) algorithm. 


$$
\mathbf{X}_{i}(k)=\left[\mathbf{x}_{i}(k), \mathbf{x}_{i}(k-1), \cdots, \mathbf{x}_{i}(k-P+1)\right],
$$

and:

$$
\mathbf{d}_{i, D}(k)=\left[d_{i, D}(k), \cdots, d_{i, D}(k-P+1)\right]^{T} .
$$

The parameter $P$ is the number of recent regressors. The IMSAF algorithm is derived from the solution of the following constraint minimization problem:

$$
\begin{aligned}
\boldsymbol{\Theta}(k)= & \|\mathbf{w}(k+1)-\mathbf{w}(k)\|^{2}+\sum_{i=0}^{N-1} \boldsymbol{\Lambda}_{i}\left[\mathbf{d}_{i, D}(k)\right. \\
& \left.-\mathbf{X}_{i}^{T}(k) \mathbf{w}(k+1)\right],
\end{aligned}
$$

where $\boldsymbol{\Lambda}_{i}=\left[\lambda_{i, 1}, \lambda_{i, 2}, \cdots, \lambda_{i, P}\right]$ is the Lagrange multipliers vector with length $P$. Using $\frac{\partial \Theta(k)}{\partial \mathbf{w}(k+1)}=0$ and $\frac{\partial \boldsymbol{\Theta}(k)}{\partial \boldsymbol{\Lambda}_{i}}=0$, we get:

$$
\mathbf{w}(k+1)=\mathbf{w}(k)+\frac{1}{2} \sum_{i=0}^{N-1} \mathbf{X}_{i}(k) \boldsymbol{\Lambda}_{i}^{T},
$$

where:

$$
\mathbf{\Lambda}_{i}^{T}=2\left[\mathbf{X}_{i}^{T}(k) \mathbf{X}_{i}(k)\right]^{-1} \mathbf{e}_{i, D}(k),
$$

and:

$$
\mathbf{e}_{i, D}(k)=\mathbf{d}_{i, D}(k)-\mathbf{X}_{i}^{T}(k) \mathbf{w}(k) .
$$

Therefore, the update equation for IMSAF becomes:

$\mathbf{w}(k+1)=\mathbf{w}(k)+\mu \sum_{i=0}^{N-1} \mathbf{X}_{i}(k)\left[\mathbf{X}_{i}^{T}(k) \mathbf{X}_{i}(k)\right]^{-1} \mathbf{e}_{i, D}(k)$.

To take care of the possibility that $\left[\mathbf{X}_{i}^{T}(k) \mathbf{X}_{i}(k)\right]$ may be close to singular, it is replaced by $\left[\epsilon \mathbf{I}+\mathbf{X}_{i}^{T}(k) \mathbf{X}_{i}(k)\right]$, where $\epsilon$ is the regularization parameter. Note that for that for $P=1$, the conventional MSAF is established. Also, it is important to note that relation in Eq. (18) is established when the cross-correlation between different sub-bands, $\left[\mathbf{X}_{i}^{T}(k) \mathbf{X}_{j}(k)\right]$, is ignored. This algorithm is called Simplified IMSAF (SIMSAF) algorithm. In the following, we use IMSAF algorithm based on this assumption [27].

\section{The SPU-IMSAF algorithm}

In this section, the SPU-IMSAF algorithm is introduced. In the SPU-IMSAF, the filter coefficients are partially updated rather than the entire filter at each subband for every adaptation. This strategy leads to a reduction in computational complexity. The SPUIMSAF algorithm is established for single and multiple blocks.

\subsection{Single block}

In the SPU-IMSAF, the filter coefficients and the input signal matrix are partitioned into the $B$ blocks each with the length $L(B=M / L$ and is an integer $)$ as follows:

$$
\mathbf{w}(k)=\left[\mathbf{w}_{1}^{T}(k), \mathbf{w}_{2}^{T}(k), \cdots, \mathbf{w}_{B}^{T}(k)\right]^{T},
$$

and:

$$
\mathbf{X}_{i}(k)=\left[\begin{array}{c}
\mathbf{X}_{i, 1}(k) \\
\mathbf{X}_{i, 2}(k) \\
\vdots \\
\mathbf{X}_{i, B}(k)
\end{array}\right]
$$

where the $L \times P$ matrices $\mathbf{X}_{i, b}(k)$ for $b=1,2, \cdots, B$ are given by Eq. (23) shown in Box I. The SPU-IMSAF minimizes the following cost function:

$$
\mathbf{\Upsilon}(k)=\min \left\|\mathbf{w}_{b}(k+1)-\mathbf{w}_{b}(k)\right\|^{2},
$$

subject to $\mathbf{X}_{i}^{T}(k) \mathbf{w}(k+1)=\mathbf{d}_{i, D}(k)$. Therefore, the cost function for the SPU-IMSAF is obtained as follows:

$$
\begin{aligned}
\mathbf{\Upsilon}(k)= & \left\|\mathbf{w}_{b}(k+1)-\mathbf{w}_{b}(k)\right\|^{2}+\sum_{i=0}^{N-1} \boldsymbol{\Lambda}_{i}\left[\mathbf{d}_{i, D}(k)\right. \\
& \left.-\mathbf{X}_{i}^{T}(k) \mathbf{w}(k+1)\right],
\end{aligned}
$$

where $\boldsymbol{\Lambda}_{i}=\left[\lambda_{i, 1}, \lambda_{i, 2}, \cdots, \lambda_{i, P}\right]$ is the Lagrange multipliers vector with length $P$. Using $\frac{\partial \boldsymbol{\Upsilon}(k)}{\partial \mathbf{w}_{b}(k+1)}=0$ and $\frac{\partial \boldsymbol{\Upsilon}(k)}{\partial \boldsymbol{\Lambda}_{i}}=0$, we get:

$$
\mathbf{w}_{b}(k+1)=\mathbf{w}_{b}(k)+\frac{1}{2} \sum_{i=0}^{N-1} \mathbf{X}_{i, b}(k) \boldsymbol{\Lambda}_{i}^{T},
$$

and:

$$
\mathbf{\Lambda}_{i}^{T}=2\left[\mathbf{X}_{i, b}^{T}(k) \mathbf{X}_{i, b}(k)\right]^{-1} \mathbf{e}_{i, D}(k) .
$$

By substituting Eq. (27) into Eq. (26), we obtain the SPU-IMSAF algorithm as follows:

$$
\begin{aligned}
\mathbf{w}_{b}(k+1)= & \mathbf{w}_{b}(k)+\mu \sum_{i=0}^{N-1} \mathbf{X}_{i, b}(k)[\epsilon \mathbf{I} \\
& \left.+\mathbf{X}_{i, b}^{T}(k) \mathbf{X}_{i, b}(k)\right]^{-1} \mathbf{e}_{i, D}(k) .
\end{aligned}
$$

It is important to note that the relation in Eq. (27)

$$
\mathbf{X}_{i, b}(k)=\left(\begin{array}{cccc}
x_{i}(k N-(b-1) L) & x_{i}(k N-(b-1) L-1) & \cdots & x_{i}(k N-(b-1) L-P+1) \\
x_{i}(k N-(b-1) L-1) & x_{i}(k N-(b-1) L-2) & \cdots & x_{i}(k N-(b-1) L-P) \\
\vdots & \vdots & \ddots & \vdots \\
x_{i}(k N-(b-1) L-L+1) & x_{i}(k N-(b-1) L-L) & \cdots & \ldots x_{i}(k N-(b-1) L-L-P+2)
\end{array}\right)(23)
$$


is established when the cross-correlation between different subbands, $\left[\mathbf{X}_{i, b}^{T}(k) \mathbf{X}_{j, b}(k)\right]$, is ignored. This phenomenon is achieved for the filter banks with good band partitioning in different subbands. Now, we turn our attention to determination of the block to be updated at each subband for every adaptation. Based on Eq. (24), the block to be updated should be selected by determining the one with the smallest squared Euclidean norm update. Therefore, by using Eq. (28), the block to be updated at each subband for every iteration is given by:

$$
\begin{aligned}
b & =\arg \min \left\|\mathbf{w}_{b}(k+1)-\mathbf{w}_{b}(k)\right\|^{2} \\
& =\arg \min \sum_{i=0}^{N-1} \mathbf{e}_{i, D}^{T}(k)\left[\mathbf{X}_{i, b}^{T}(k) \mathbf{X}_{i, b}(k)\right]^{-1} \mathbf{e}_{i, D}(k) .
\end{aligned}
$$

Since the computational complexity of Eq. (29) is high, a simplified approach to finding the index of the block at each subband for every adaptation is proposed as in the following $[8,11,16]$ :

1. Compute the following values for $1 \leq b \leq B$ :

$$
\sum_{i=0}^{N-1} \operatorname{Tr}\left[\mathbf{X}_{i, b}^{T}(k) \mathbf{X}_{i, b}(k)\right] .
$$

2. The index $b$ at each subband corresponds to the largest value of Eq. (30).

\subsection{Extension to multiple blocks}

Suppose that we wish to update $Q$ blocks out of $B$ at each subband for every iteration. Let $G_{Q}=$ $\left[b_{1}, b_{2}, \cdots, b_{Q}\right]$ denote a $Q$-subset (subset with $Q$ members) of the set $\{1,2, \cdots, B\}$. The SPU-IMSAF minimizes the following cost function:

$$
\Upsilon_{G_{Q}}(k)=\min \left\|\mathbf{w}_{G_{Q}}(k+1)-\mathbf{w}_{G_{Q}}(k)\right\|^{2},
$$

subject to $\mathbf{X}_{i}^{T}(k) \mathbf{w}(k+1)=\mathbf{d}_{i, D}(k)$, where:

$$
\mathbf{w}_{G_{Q}}(k)=\left[\mathbf{w}_{b_{1}}^{T}(k), \mathbf{w}_{b_{2}}^{T}(k), \cdots, \mathbf{w}_{b_{Q}}^{T}(k)\right]^{T} .
$$

Therefore, the cost function is defined as follows:

$$
\begin{aligned}
\Upsilon_{G_{Q}}(k)= & \left\|\mathbf{w}_{G_{Q}}(k+1)-\mathbf{w}_{G_{Q}}(k)\right\|^{2} \\
& +\sum_{i=0}^{N-1} \boldsymbol{\Lambda}_{i}\left[\mathbf{d}_{i, D}(k)-\mathbf{X}_{i}^{T}(k) \mathbf{w}(k+1)\right] .
\end{aligned}
$$

Using $\frac{\partial \boldsymbol{\Upsilon}_{G_{Q}}(k)}{\partial \mathbf{w}_{G_{Q}}(k+1)}=0$ and $\frac{\partial \Upsilon_{G_{Q}}(k)}{\partial \boldsymbol{\Lambda}_{i}}=0$ leads to the following update equation:

$$
\begin{aligned}
\mathbf{w}_{G_{Q}}(k+1)= & \mathbf{w}_{G_{Q}}(k)+\mu \sum_{i=0}^{N-1} \mathbf{X}_{i, G_{Q}}(k)[\epsilon \mathbf{I} \\
& \left.+\mathbf{X}_{i, G_{Q}}^{T}(k) \mathbf{X}_{i, G_{Q}}(k)\right]^{-1} \mathbf{e}_{i, D}(k),
\end{aligned}
$$

where:

$$
\mathbf{X}_{i, G_{Q}}(k)=\left[\begin{array}{c}
\mathbf{X}_{i, b_{1}}(k) \\
\mathbf{X}_{i, b_{2}}(k) \\
\vdots \\
\mathbf{X}_{i, b_{Q}}(k)
\end{array}\right]
$$

The indices of the blocks are obtained according to the following condition:

$$
\begin{aligned}
G_{Q} & =\arg \min \left\|\mathbf{w}_{G_{Q}}(k+1)-\mathbf{w}_{G_{Q}}(k)\right\|^{2} \\
& =\arg \min \sum_{i=0}^{N-1} \mathbf{e}_{i, D}^{T}(k)\left[\mathbf{X}_{i, G_{Q}}^{T}(k) \mathbf{X}_{i, G_{Q}}(k)\right]^{-1} \mathbf{e}_{i, D}(k) .
\end{aligned}
$$

Due to the high computational complexity of Eq. (36), a simplified approach to selecting the indices of $G_{Q}$ is proposed as in the following $[8,11,16]$ :

1. Compute the following values for $1 \leq b \leq B$ :

$$
\sum_{i=0}^{N-1} \operatorname{Tr}\left[\mathbf{X}_{i, b}^{T}(k) \mathbf{X}_{i, b}(k)\right]
$$

2. The indices of $G_{Q}$ at each subband correspond to the $Q$ largest values of Eq. (37).

Table 1 summarizes the SPU-IMSAF algorithm.

\section{The SM-IMSAF Algorithm}

The SM-NLMS algorithm was introduced in [22] which had a fast convergence speed, low steady-state error, and low computational complexity features. This idea was extended to APA and MSAF in [16,23]. To improve the performance of the IMSAF, the SM method was extended to IMSAF algorithm. First, we define $\mathbf{x}_{i, D}(k)$ and $d_{i, D}(k)$ at time instant $k$ and the constraint set $\mathcal{H}_{i}(k)$ containing all vectors $\mathbf{w}$ with estimation errors upper bounded in magnitude by $\gamma$ as follows:

$$
\mathcal{H}_{i}(k)=\left\{\mathbf{w} \in \mathcal{R}^{M}:\left|d_{i, D}(k)-\mathbf{x}_{i, D}^{T}(k) \mathbf{w}\right| \leq \gamma\right\} .
$$

The membership set $\Psi_{i}(k)$ is defined as:

$$
\Psi_{i}(k)=\mathcal{H}_{i}(1) \cap \mathcal{H}_{i}(2) \cdots \cap \mathcal{H}_{i}(k) .
$$

Since $\Psi_{i}(k)$ in Eq. (39) is not easily computed, adaptive approaches are needed. For example, in SM-MSAF, the information is provided by the constraint set $\mathcal{H}_{i}(k)$ [16]. The update equation for SM-MSAF algorithm was introduced as follows:

$$
\mathbf{w}(k+1)=\mathbf{w}(k)+\sum_{i=0}^{N-1} \alpha_{i}(k) \frac{\mathbf{x}_{i}(k)}{\left\|\mathbf{x}_{i}(k)\right\|^{2}} e_{i, D}(k),
$$

where:

$$
\alpha_{i}(k)= \begin{cases}1-\frac{\gamma}{\left|e_{i, D}(k)\right|} & \text { if }\left|e_{i, D}(k)\right|>\gamma \\ 0 & \text { otherwise }\end{cases}
$$

In SM-MSAF, the filter coefficients are updated ac- 
Table 1. The SPU-IMSAF algorithm.

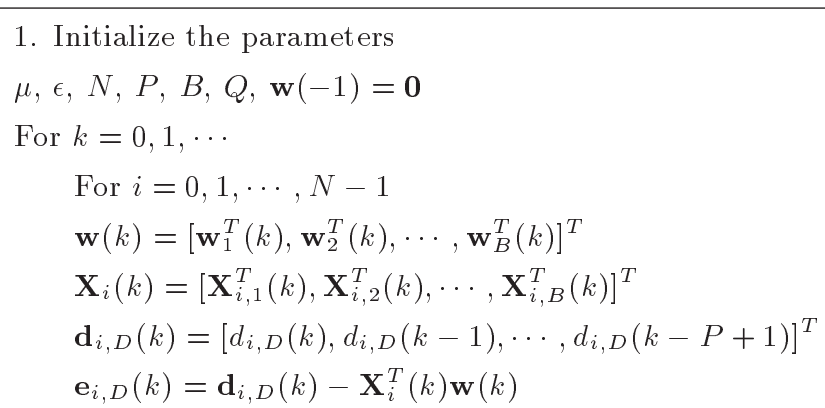

2. Determine the indices of $G_{Q}=\left[b_{1}, b_{2}, \cdots, b_{Q}\right]$ according to $Q$ largest values of $(\star)$

For $1 \leq b \leq B$

$\sum_{i=0}^{N-1} \operatorname{Tr}\left[\mathbf{X}_{i, b}^{T}(k) \mathbf{X}_{i, b}(k)\right](\star)$

end

3. Update the input signal matrix according to the selected blocks

$$
\mathbf{X}_{i, G_{Q}}(k)=\left[\mathbf{X}_{i, b_{1}}^{T}(k), \cdots, \mathbf{X}_{i, b_{Q}}^{T}(k)\right]^{T}
$$

4. Select the blocks of filter coefficients according to the selected blocks

$$
\mathbf{w}_{G_{Q}}=\left[\mathbf{w}_{b_{1}}^{T}(k), \mathbf{w}_{b_{2}}^{T}(k), \cdots, \mathbf{w}_{b_{Q}}^{T}(k)\right]^{T}
$$

5. Update the filter coefficients

$$
\begin{aligned}
& \mathbf{w}_{G_{Q}}(k+1)=\mathbf{w}_{G_{Q}}(k)+\mu \sum_{i=0}^{N-1} \mathbf{X}_{i, G_{Q}}(k)\left[\epsilon \mathbf{I}+\mathbf{X}_{i, G_{Q}}^{T}(k) \mathbf{X}_{i, G_{Q}}(k)\right]^{-1} \mathbf{e}_{i, D}(k) \\
& \text { End }
\end{aligned}
$$

cording to the condition in Eq. (41). If the condition is satisfied, the filter coefficients are updated. Therefore, the number of filter coefficients in the update coefficient is reduced. In the following, we derive the SM-IMSAF whose updates that belong to a set formed by $P$ constraint sets. Let $\Psi_{i}^{P}(k)$ be the intersection of the last constraint sets as follows [23]:

$$
\Psi_{i}^{P}(k)=\mathcal{H}_{i}(k-P+1) \cap \cdots \mathcal{H}_{i}(k) .
$$

The objective is to derive an algorithm whose updated filter coefficients belong to the last $P$ constraint-sets, $\mathbf{w}(k+1) \in \Psi_{i}^{P}(k)$. The SM-IMSAF minimizes Eq. (12) subject to:

$$
\mathbf{d}_{i, D}(k)-\mathbf{X}_{i}^{T}(k) \mathbf{w}(k+1)=\mathbf{g}_{i}(k),
$$

where:

$$
\mathbf{g}_{i}(k)=\left[g_{i}(k), g_{i}(k-1), \cdots, g_{i}(k-P+1)\right]^{T},
$$

specifies the point in $\Psi_{i}^{P}(k)$. All choices for $\mathbf{g}_{i}(k)$ satisfying the bound constraint are valid. By using the method of Lagrange multipliers, the following cost function is introduced as follows:

$$
\begin{aligned}
\boldsymbol{\Omega}(k)= & \|\mathbf{w}(k+1)-\mathbf{w}(k)\|^{2}+\sum_{i=0}^{N-1} \boldsymbol{\Lambda}_{i}\left[\mathbf{d}_{i, D}(k)\right. \\
& \left.-\mathbf{X}_{i}^{T}(k) \mathbf{w}(k+1)-\mathbf{g}_{i}(k)\right] .
\end{aligned}
$$

Solving this optimization problem leads to:

$$
\begin{aligned}
\mathbf{w}(k+1)= & \mathbf{w}(k)+\sum_{i=0}^{N-1} \mathbf{X}_{i}(k)\left[\mathbf{X}_{i}^{T}(k) \mathbf{X}_{i}(k)\right]^{-1} \\
& \times\left[\mathbf{e}_{i, D}(k)-\mathbf{g}_{i}(k)\right] .
\end{aligned}
$$

Eq. (46) is performed when $\left|e_{i, D}(k)\right|>\gamma$ and otherwise, the filter coefficients do not change. There are several choices for $\mathbf{g}_{i}(k)$. The simplest choice is $\mathbf{g}_{i}(k)=\mathbf{0}$. This approach leads to a considerable complexity reduction in comparison with conventional IMSAF. Another choice is $g_{i}(k)=\gamma \operatorname{sgn}\left(e_{i, D}(k)\right)$ [23]. In this case, the update equation is given by:

$$
\begin{aligned}
\mathbf{w}(k+1)= & \mathbf{w}(k)+\sum_{i=0}^{N-1} \mathbf{X}_{i}(k)[\epsilon \mathbf{I} \\
& \left.+\mathbf{X}_{i}^{T}(k) \mathbf{X}_{i}(k)\right]^{-1} \alpha_{i}(k) e_{i, D}(k) \mathbf{u}_{1},
\end{aligned}
$$

where $\mathbf{u}_{1}=[1,0,0, \cdots, 0]^{T}$ is $P \times 1$ vector and $\alpha_{i}(k)$ is obtained by Eq. (41). Since Eq. (47) is related to the first element of the error vector at each subband, the following update equation for SM-IMSAF is introduced as follows:

$$
\begin{aligned}
\mathbf{w}(k+1)= & \mathbf{w}(k)+\mu \sum_{i=0}^{N-1} \alpha_{i}(k) \mathbf{X}_{i}(k)[\epsilon \mathbf{I} \\
& \left.+\mathbf{X}_{i}^{T}(k) \mathbf{X}_{i}(k)\right]^{-1} \mathbf{e}_{i, D}(k),
\end{aligned}
$$

where: 


$$
\alpha_{i}(k)= \begin{cases}1-\frac{\gamma}{\left\|\mathbf{e}_{i, D}(k)\right\|_{2}} & \text { if }\left\|\mathbf{e}_{i, D}(k)\right\|_{2}>\gamma \\ 0 & \text { otherwise }\end{cases}
$$

In Eq. (48), the step-size controls the stability of the algorithm. Since $\mathbf{e}_{i, D}(k)$ is the vector, we use an Euclidean norm operator $\left(L_{2}\right.$-norm) in Eq. (49). We compared the learning curves based on $L_{2}$-norm and $L_{1}$-norm of $\mathbf{e}_{i, D}(k)$ in the simulation results section. Table 2 summarizes the SM-IMSAF algorithm. By combining the SPU and SM approaches, the SM-SPUIMSAF can be established. The update equation for SM-SPU-IMSAF is proposed as follows:

$$
\begin{aligned}
\mathbf{w}_{G_{Q}}(k+1)= & \mathbf{w}_{G_{Q}}(k)+\mu \sum_{i=0}^{N-1} \alpha_{i}(k) \mathbf{X}_{i, G_{Q}}(k)[\epsilon \mathbf{I} \\
& \left.+\mathbf{X}_{i, G_{Q}}^{T}(k) \mathbf{X}_{i, G_{Q}}(k)\right]^{-1} \mathbf{e}_{i, D}(k) .
\end{aligned}
$$

In this algorithm, the filter coefficients are partially updated and the subbands are selected according to Eq. (49) at each iteration. Combining these strategies significantly reduces the computational complexity.

\section{Computational complexity}

Table 3 compares the computational complexity of the IMSAF, SM-IMSAF, SPU-IMSAF, and SM-SPUIMSAF algorithms in terms of the number of multiplications per iteration for real data. In this table, $M$ is the filter length, $N$ the number of subbands, $P$ the number of input regressors, $L$ the length of channel filters, $B$ the number of blocks, $Q$ the number of selected blocks, and $N(k)$ the number of selected subbands according to Eq. (49) at iteration $k$. As we can see, the number of multiplications in SPU-IMSAF is smaller than that in IMSAF, especially for large values of $M$. In SM-IMSAF, the number of multiplications at each iteration is dependent on the condition in Eq. (49). The parameter $\alpha_{i}(k)$ determines which subband is incorporated into the update equation. In the worst case, the computational complexity of SM-IMSAF is the same as IMSAF. It means that all subbands will be selected at each iteration. In the simulation results section, we show that the computational complexity of SM-IMSAF is significantly lower than IMSAF. We also observe that SM-SPU-IMSAF has lower computational

Table 2. The SM-IMSAF algorithm.

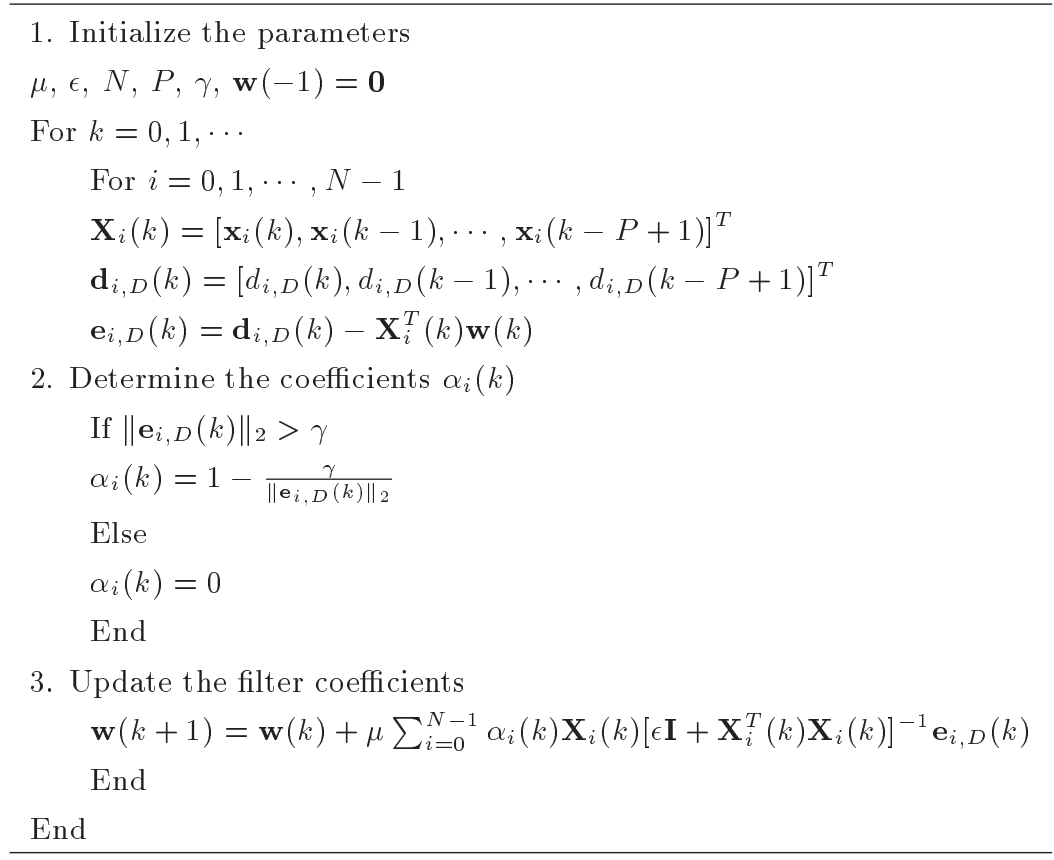

Table 3. Computational complexity of Improved Multiband-Structured Subband Adaptive Filter (IMSAF), SM-IMSAF, SPU-IMSAF, and SM-SPU-IMSAF algorithms per iteration.

\begin{tabular}{lc}
\hline \multicolumn{1}{c}{ Algorithm } & Number of multiplications \\
\hline IMSAF & $\left(P^{2}+2 P\right) M+P^{3}+P^{2}+3 N L$ \\
SM-IMSAF & $\frac{N(k)}{N}\left[\left(P^{2}+2 P\right) M+P^{3}+P^{2}+1\right]+P+3 N L$ \\
SPU-IMSAF & $M P+\left(P^{2}+2 P\right) Q L+P^{3}+P^{2}+3 N L$ \\
SM-SPU-IMSAF & $P(M+1)+\frac{N(k)}{N}\left[\left(P^{2}+2 P\right) Q L+P^{3}+P^{2}+1\right]+3 N L$ \\
\hline
\end{tabular}


complexity than SM-IMSAF due to SM and SPU strategies.

\section{Simulation results}

We demonstrate the performance of the proposed algorithm by several computer simulations in Acoustic Echo Cancellation (AEC) setup. The impulse response of the car echo path with 256 taps $(M=256)$ was used as an unknown system in the experiment (Figure 2) [11]. The input signal is an $\operatorname{AR}(1)$ signal generated by passing a zero-mean white Gaussian noise with unit variance through a first-order system $H(z)=\frac{1}{1-0.95 z^{-1}}$ and the value of $\sigma_{v}^{2}$ was set to $10^{-2}$. The filter bank used in the simulations was the Extended Lapped Transform (ELT) [16,28]. In all simulations, we show the Normalized Mean Square Deviation (NMSD), $E\left[\frac{\left\|\mathbf{w}^{\circ}-\mathbf{w}(k)\right\|^{2}}{\left\|\mathbf{w}^{\circ}\right\|^{2}}\right]$, which is evaluated by ensemble averaging over 50 independent trials.

Since the exact IMSAF algorithm is characterized by large computational complexity [24], the simplified version of this algorithm was applied in the literature [27]. Therefore, we firstly compare the performance of IMSAF and SIMSAF algorithms in Figure 3. The parameter $P$ is set to 4 and two values for $N$ are selected. The step-size is set to 0.5 for both values of $N$. We observe that the SIMSAF has close performance to IMSAF algorithm. In the following, we use the same name (IMSAF) for both algorithms. Figure 4 compares the NMSD learning curves of SPU-IMSAF algorithm (single block) based on Eqs. (29) and (30). The stepsize is set 0.05 and the parameter $N$ is set to 2,4 , and 8 . Also, the parameters $P$ and $B$ are set to 4 . We observe that the approximation relation (Eq. (30)) has close performance to the exact relation (Eq. (29)). Figure 5 presents the same results for multiple blocks situation with $Q=3$. In this case, the step-size is set to 0.3 . Again, close performance between the learning curves

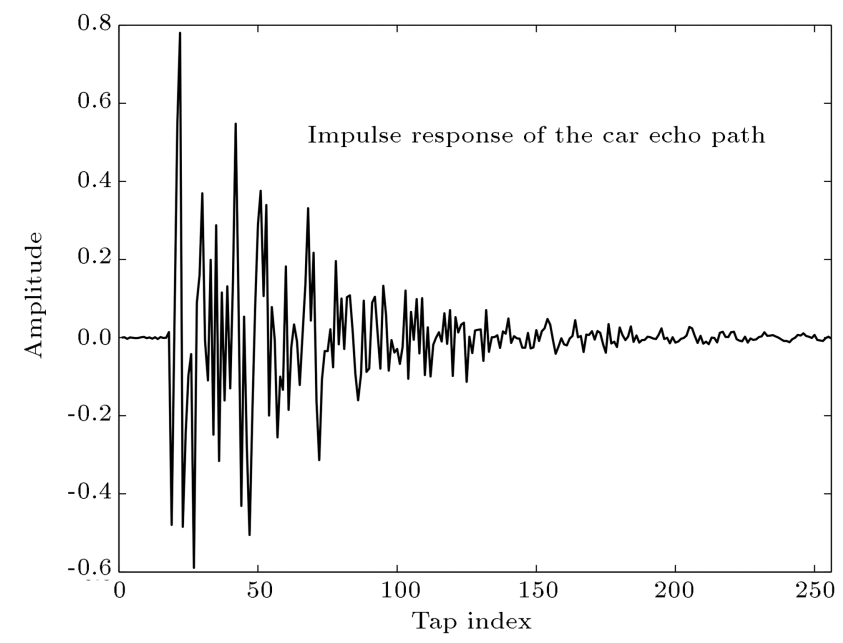

Figure 2. Impulse responses of car echo paths.

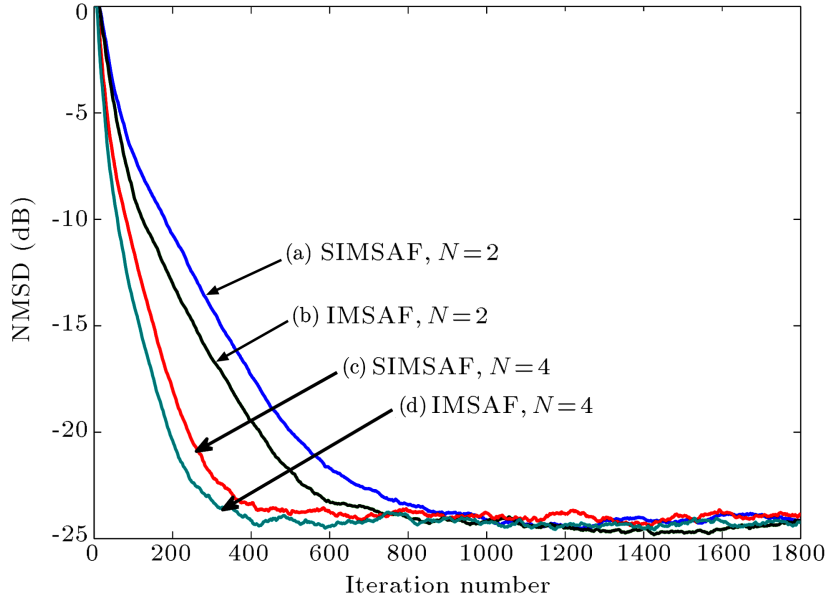

Figure 3. The Normalized Mean Square Deviation (NMSD) learning curves of Improved Multiband-Structured Subband Adaptive Filter (IMSAF) and simplified IMSAF (SIMSAF) for $N=2$ and 4 $(M=256, P=4, \mu=0.5)$.

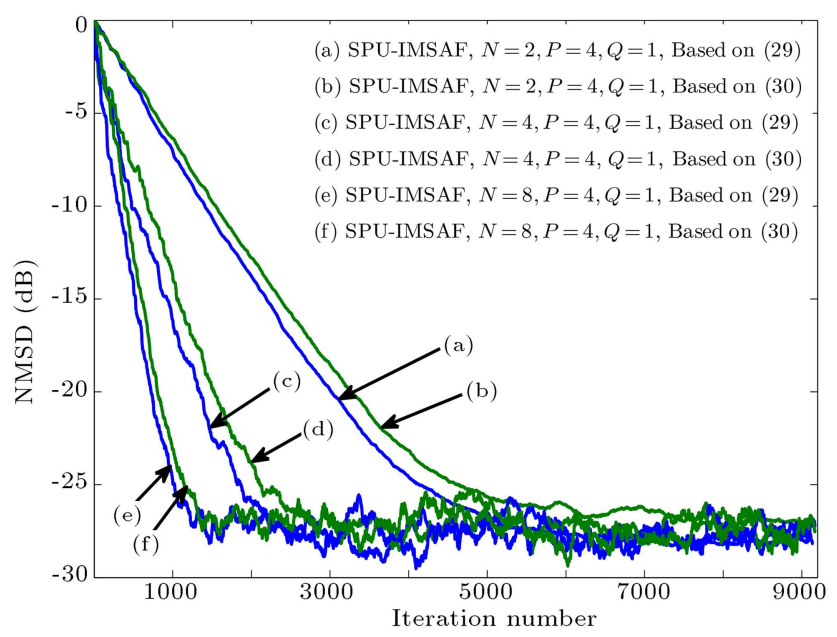

Figure 4. The Normalized Mean Square Deviation (NMSD) learning curves of SPU-IMSAF based on Eqs. (29) and (30).

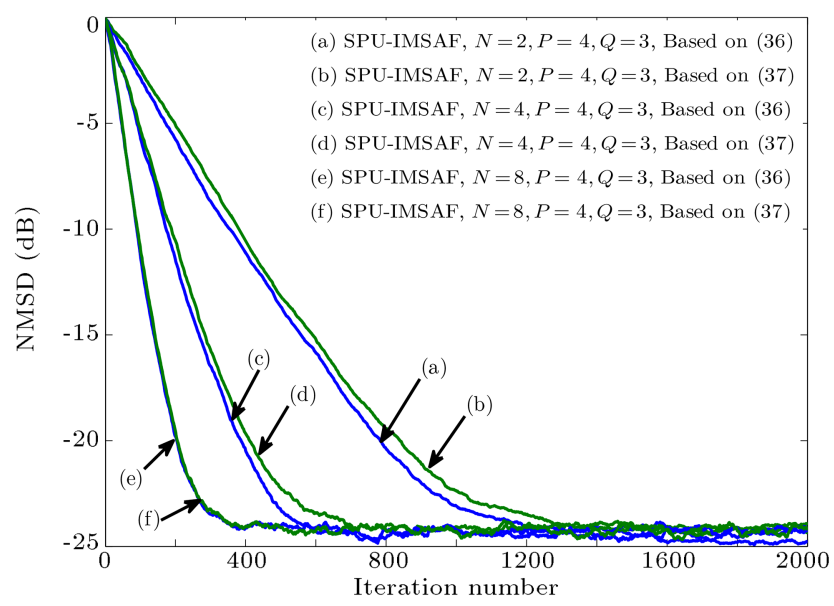

Figure 5. The Normalized Mean Square Deviation (NMSD) learning curves of SPU-IMSAF based on Eqs. (36) and (37). 


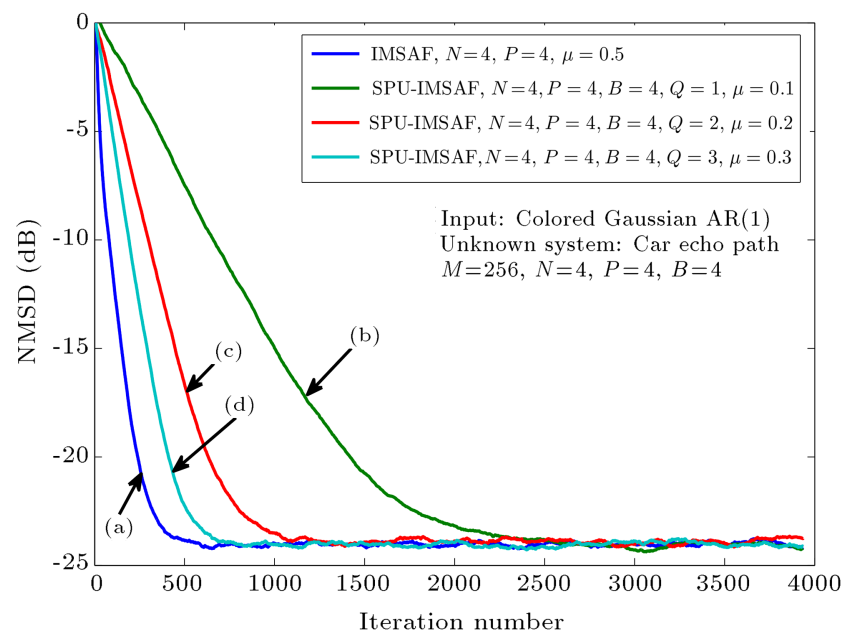

Figure 6. The Normalized Mean Square Deviation (NMSD) learning curves of the Improved

Multiband-Structured Subband Adaptive Filter (IMSAF) and the proposed SPU-IMSAF algorithms for the same steady-state error $(M=256, N=4, B=4)$.

can be seen based on Eqs. (36) and (37) for different values of $N$.

Figure 6 presents the NMSD learning curves of IMSAF and SPU-IMSAF algorithms with $N=4$. The parameters $N$ and $P$ are set to 4 . In SPU-IMSAF, the number of blocks $(B)$ is set to 4 and different values for $Q$ are chosen. Also, the step-size in IMSAF is set to 0.5 and to make the comparison fair, the step-size for SPUIMSAF algorithm is chosen to get approximately the same steady-state NMSD as IMSAF. The results show that by increasing the parameter $Q$, the performance of SPU-IMSAF will be close to the IMSAF. For $Q=$ 3 , the similar performance between IMSAF and SPUIMSAF is observed. It is important to note that for SPU-IMSAF with $Q=2$ and $Q=3,128$ and 192 coefficients out of 256 are updated.

In Figures 7 and 8, we set the number of blocks $(B)$ to 16 . Figure 7 shows the learning curve for different values of $Q$. In this simulation, the stepsize is set to 0.5 . By increasing the parameter $Q$, the performance of SPU-IMSAF will be close to IMSAF. The NMSD learning curves for the same steady-state error are shown in Figure 8. The step-size in IMSAF is set to 0.5 and to make the comparison fair, the stepsize for SPU-IMSAF algorithm is chosen to get approximately the same steady-state NMSD as IMSAF. The values of the step-size for $Q=10,12,14$, and 16 are $0.26,0.33,0.4$, and 0.5 , respectively. The results show that the performance of SPU-IMSAF algorithm for $Q=10,12$, and 14 is close to the conventional IMSAF algorithm. Figure 9 compares the performance of the SPU-IMSAF algorithm for different values of $B$ and $Q$ where the ratio, $Q / B$, is fixed. In this case, the number of filter coefficients updated at each iteration is 192. We observe close performance for all algorithms.

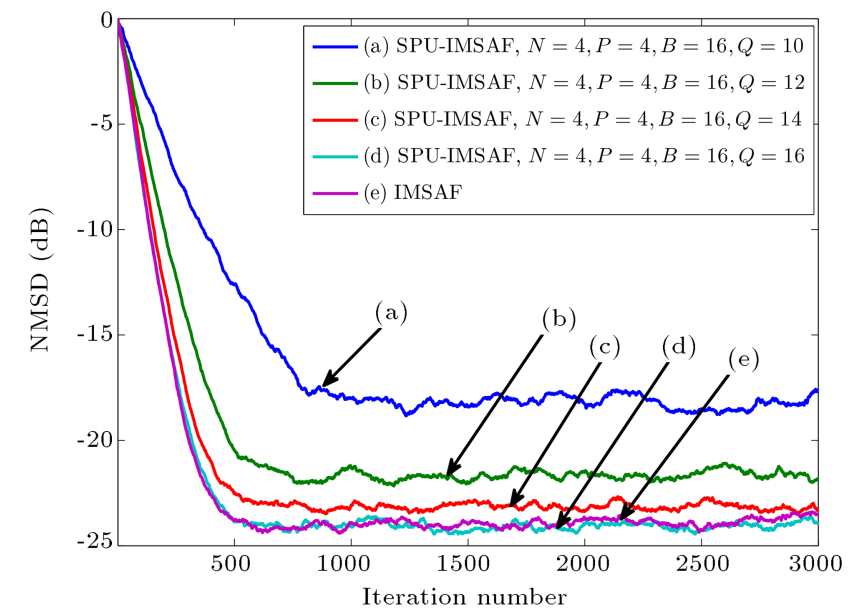

Figure 7. The Normalized Mean Square Deviation (NMSD) learning curves of the Improved Multiband-Structured Subband Adaptive Filter (IMSAF) and the proposed SPU-IMSAF algorithms $(M=256$, $N=4, B=16, \mu=0.5$ ).

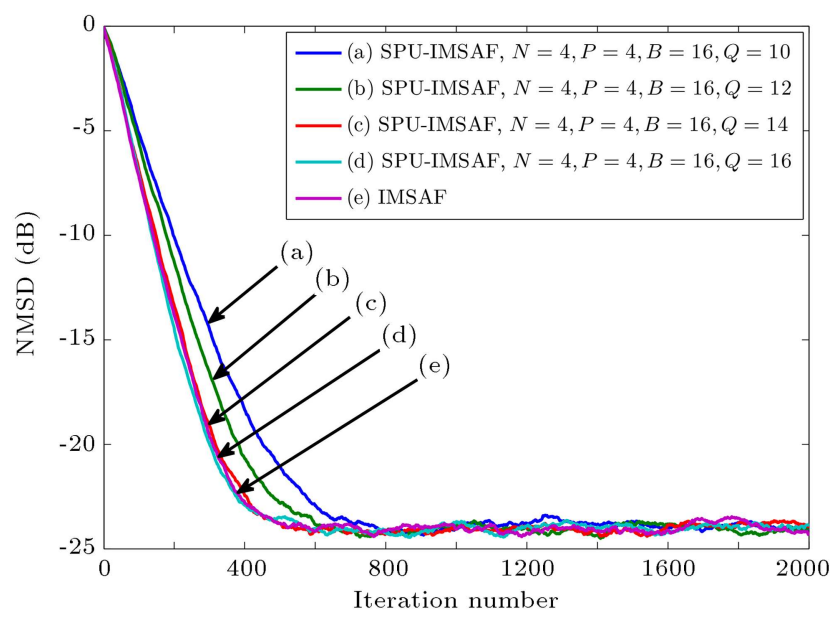

Figure 8. The Normalized Mean Square Deviation (NMSD) learning curves of the Improved Multiband-Structured Subband Adaptive Filter (IMSAF) and the proposed SPU-IMSAF algorithms for the same steady-state error $(M=256, N=4, B=16)$.

The performance of SPU-IMSAF for $B=16$ and $Q=12$ is slightly better than other curves. Figure 10 presents the steady-state NMSD values versus $Q$ for different values of $N$. The parameters $B$ and $P$ were set to 4. Also, the step-size was set 0.1. As we can see, by increasing the parameter $Q$, the steady-state NMSD decreases. This observation can be seen for $B=16$ in Figure 11.

Figure 12 shows the NMSD learning curves of SMIMSAF algorithm based on $L_{1}$ and $L_{2}$ norms of $\mathbf{e}_{i, D}(k)$. The parameters $P$ and $N$ are set to 4 and the step-size is set 0.5. We observe that the performance of SMIMSAF based on $L_{2}$-norm of $\mathbf{e}_{i, D}(k)$ is better than $L_{1}$-norm. This advantage can be seen in Figure 13 for $N=8$. Therefore, in the following, we use $L_{2^{-}}$ 


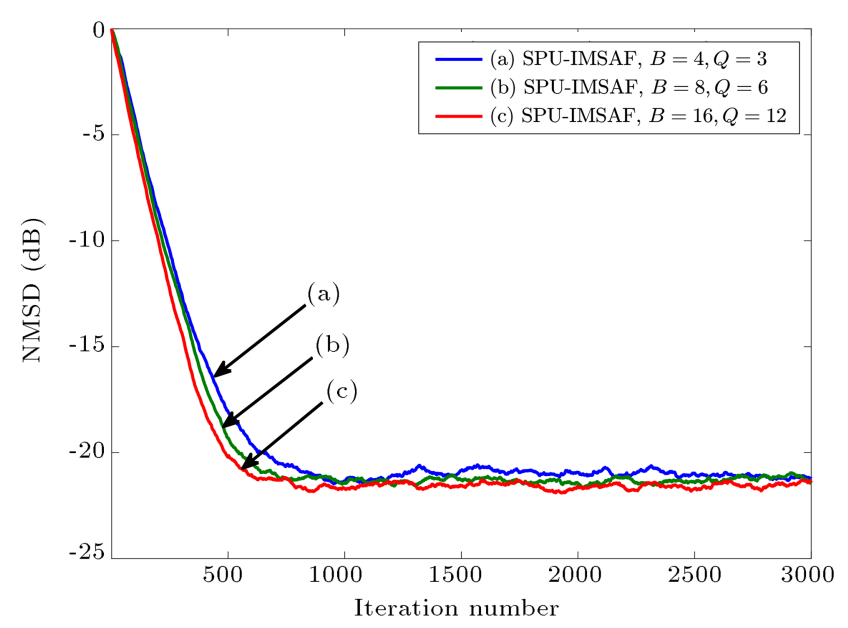

Figure 9. The Normalized Mean Square Deviation (NMSD) learning curves of SPU-IMSAF with different values of $B$ and $Q$.

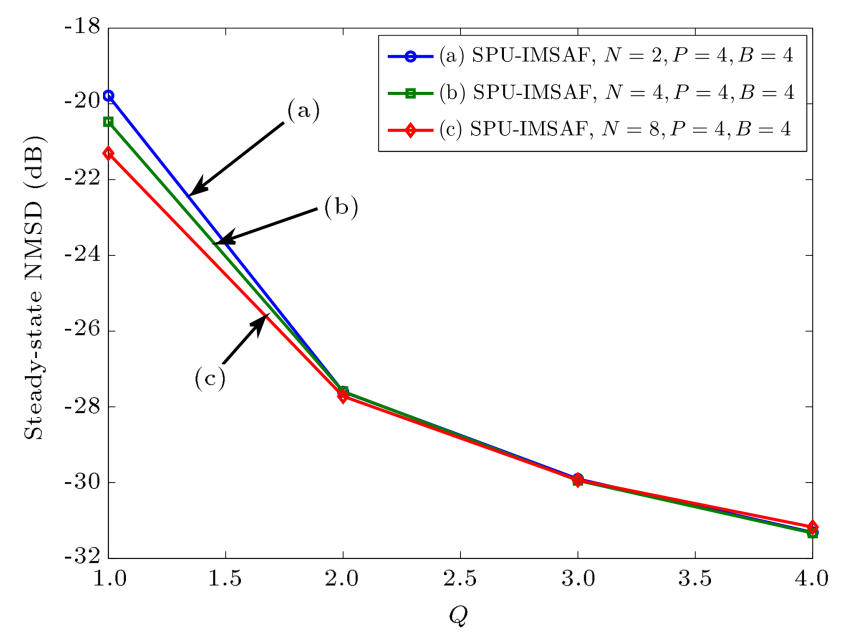

Figure 10. The steady-state Normalized Mean Square Deviation (NMSD) versus $Q$ with different values of $N$ for SPU-IMSAF ( $M=256, B=4, \mu=0.1)$.

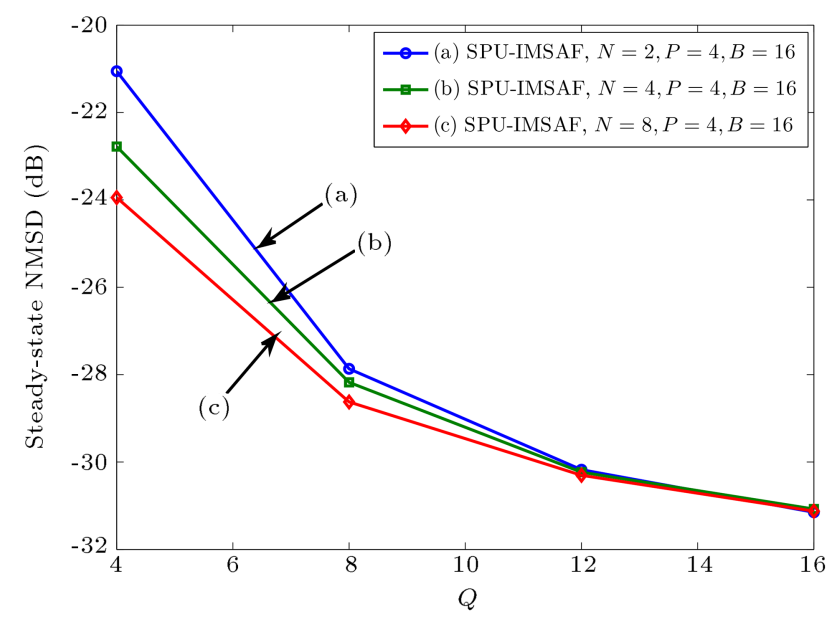

Figure 11. The steady-state Normalized Mean Square Deviation (NMSD) versus $Q$ with different values of $N$ for SPU-IMSAF $(M=256, B=16, \mu=0.1)$.

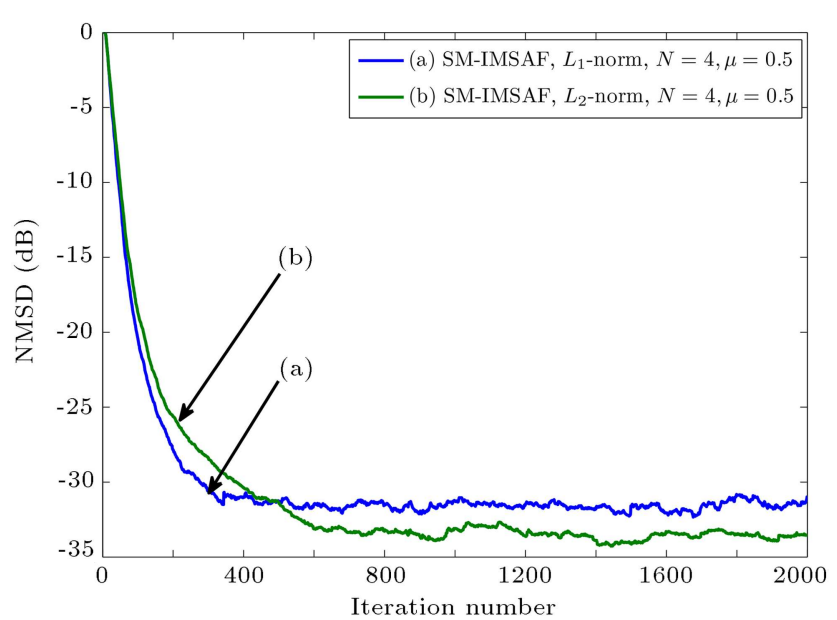

Figure 12. The Normalized Mean Square Deviation (NMSD) learning curves of SM-IMSAF based on $L_{1}$-norm and $L_{2}$-norm of $\mathbf{e}_{i, D}(k)(M=256, N=4, \mu=0.5)$.

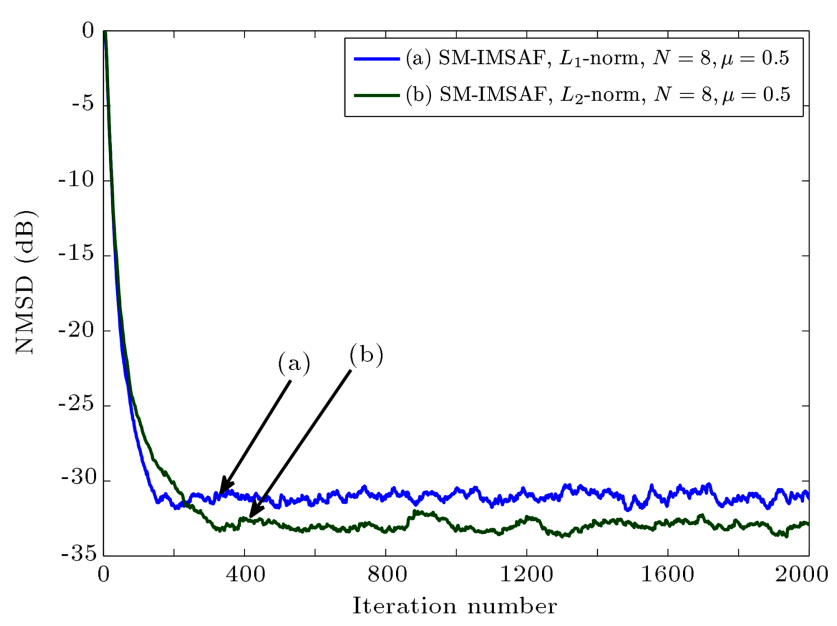

Figure 13. The Normalized Mean Square Deviation (NMSD) learning curves of SM-IMSAF based on $L_{1}$-norm and $L_{2}$-norm of $\mathbf{e}_{i, D}(k)(M=256, N=8, \mu=0.5)$.

norm of $\mathbf{e}_{i, D}(k)$. Figure 14 compares the performance of IMSAF and SM-IMSAF for $N=4$ and 8. For IMSAF, two values for the step-size were chosen $(0.1$ and 0.5$)$. In SM-IMSAF, the value of $\gamma$ was set to $\sqrt{5 \sigma_{v}^{2}}[16,23]$. The results show that SM-IMSAF has better performance than IMSAF. Figure 15 shows that when the filter coefficients at each subband $(i=$ $0,1,2,3)$ are updated during the adaptation. This figure shows that at different iterations, we do not need to update the filter coefficients which can, in turn, reduce computational complexity. Figure 16 shows these selections in the limited range of iterations. We clearly observe that the adaptation is not performed at different subbands.

Figure 17 compares the performance of IMSAF with all the proposed algorithms. This figure shows that the SPU-IMSAF has close performance to IMSAF. Also, the SM-IMSAF and SM-SPU-IMSAF algorithms 

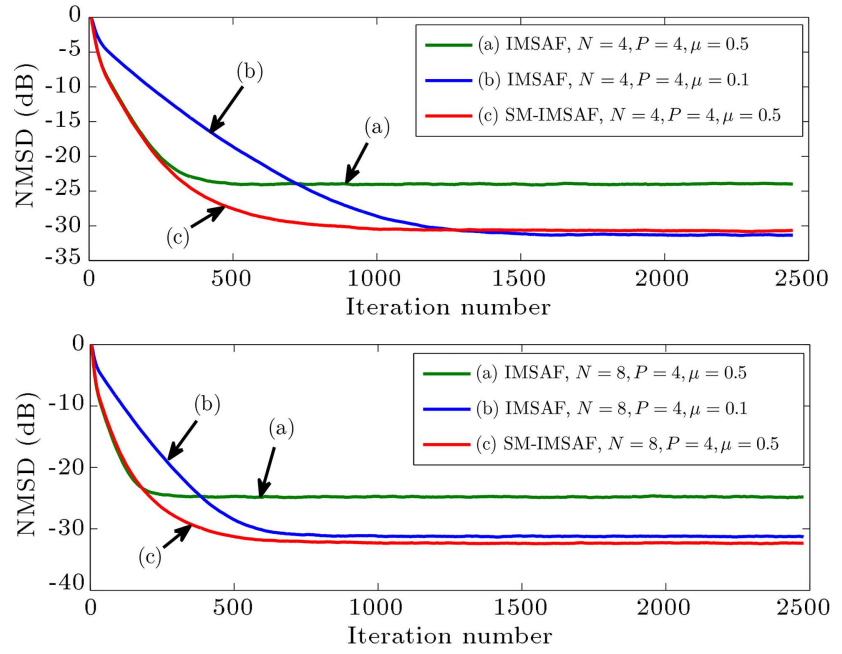

Figure 14. The Normalized Mean Square Deviation (NMSD) learning curves of the Improved

Multiband-Structured Subband Adaptive Filter (IMSAF) and the proposed SM-IMSAF algorithms $(M=256$, $P=4$, and $N=4,8)$.
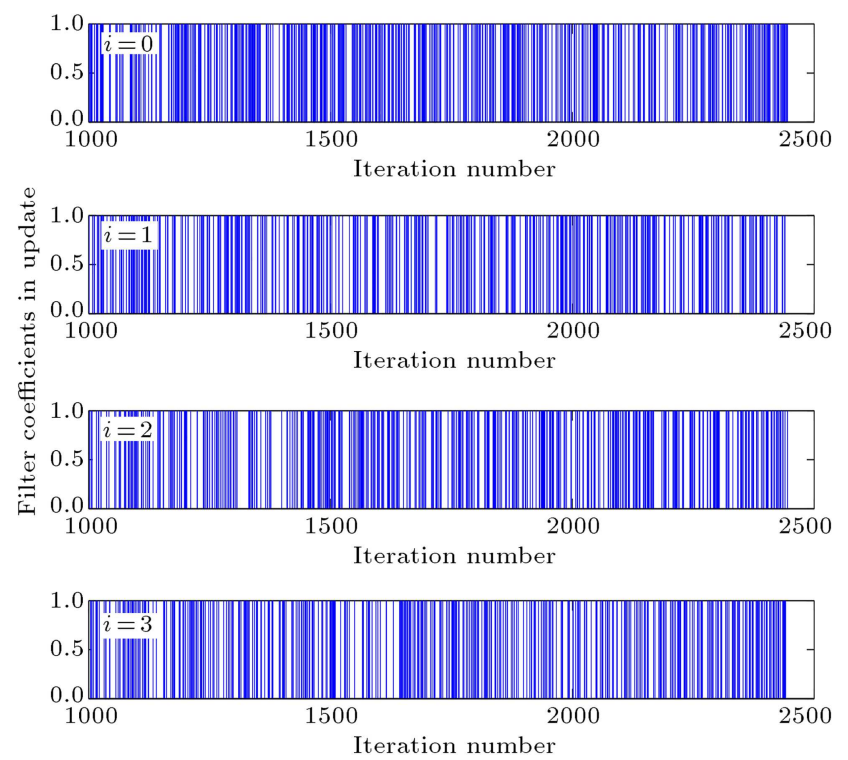

Figure 15. Filter coefficients in update for SM-IMSAF at different subbands $(M=256, N=4, P=4)$.

have faster convergence speed and lower steady-state error than conventional IMSAF. Figure 18 compares the performance of the proposed algorithms with those of SR-IMSAF and DSR-IMSAF algorithms in [27] for the same steady-state error. We observe that the SMIMSAF has better convergence speed than other algorithms. Also, SM-SPU-IMSAF has better performance than IMSAF and close to the DSR-IMSAF algorithm. Table 4 shows the total number of multiplications, the processing time, and the number of iterations until convergence is achieved based on Figure 18. As is observed, the computational complexity of the proposed algorithms is lower than that of IMSAF. The SM-
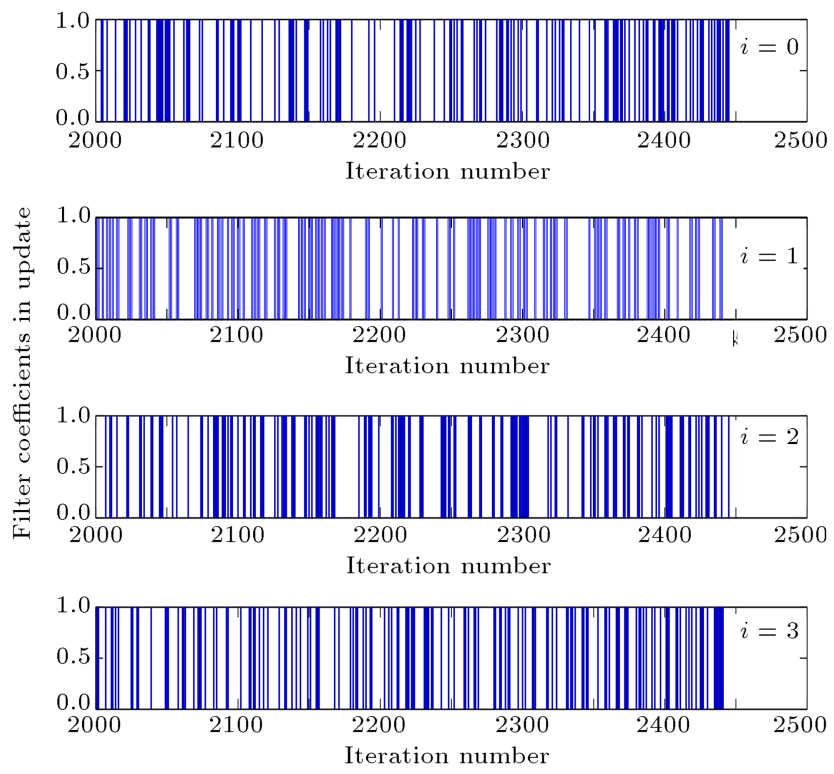

Figure 16. Filter coefficients in update for SM-IMSAF at different subbands in the limited range of iterations $(M=256, N=4, P=4)$.

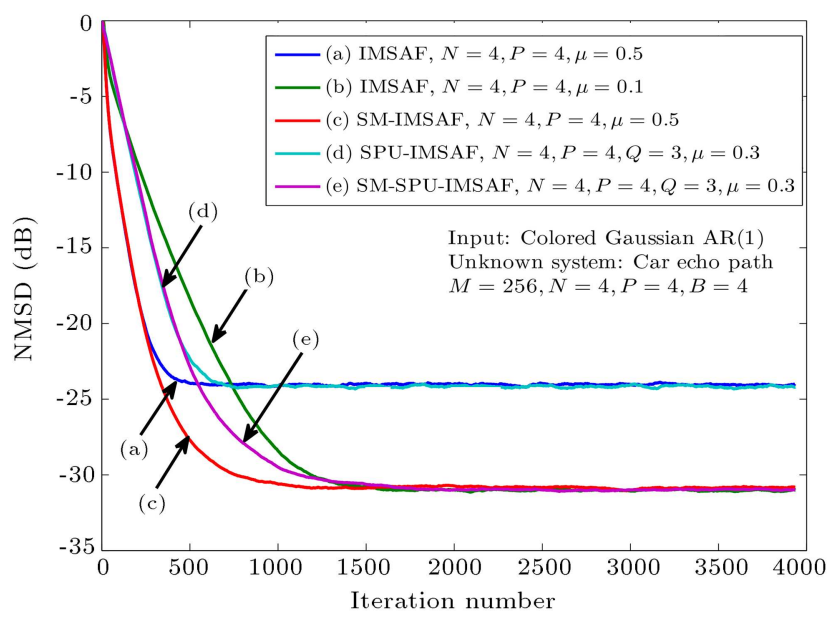

Figure 17. The Normalized Mean Square Deviation (NMSD) learning curves of Improved

Multiband-Structured Subband Adaptive Filter (IMSAF) and proposed SPU-IMSAF, SM-IMSAF, and

SM-SPU-IMSAF algorithms $(M=256, N=4, P=4$, $B=4, Q=3)$.

IMSAF needs $3.88 \times 10^{6}$ multiplications. In the worst case, the number of multiplications of SM-IMSAF is the same as that of IMSAF. However, the performance of SM-IMSAF is better than other algorithms in both convergence speed and steady-state error features.

Figures 19-21 show the NMSD learning curves of the proposed algorithms at different levels of Signal to Noise Ratio (SNR). The parameter $B$ is set to 4 . The values of $N$ and $P$ are set to 2 and $Q$ is set to 3 . Also, the step-size is set to 0.5. Figure 19 presents the results for IMSAF and SPU-IMSAF. We observe that by decreasing the level of SNR, the steady-state error 
Table 4. Total number of multiplications, processing time, and the number of iterations until convergence for Improved Multiband-Structured Subband Adaptive Filter (IMSAF), SR-IMSAF, DSR-IMSAF, SPU-IMSAF, SM-IMSAF, and SM-SPU-IMSAF algorithms until convergence.

\begin{tabular}{lccc}
\hline Algorithm & $\begin{array}{c}\text { Number of } \\
\text { multiplications }\end{array}$ & $\begin{array}{c}\text { Processing time } \\
\text { (sec) }\end{array}$ & $\begin{array}{c}\text { Iterations until } \\
\text { convergence }\end{array}$ \\
\hline IMSAF, $N=4, P=4, \mu=0.1$ & $3.84 \times 10^{7}$ & 60.51 & 1500 \\
SR-IMSAF, $N=4, P=4, \mu=0.1$ & $3.11 \times 10^{7}$ & 49 & 1800 \\
DSR-IMSAF, $N=4, P=4, \mu=0.15$ & $1.44 \times 10^{7}$ & 22.69 & 1300 \\
SM-IMSAF, $N=4, P=4, \mu=0.5$ & $3.88 \times 10^{6}$ & 6.11 & 1000 \\
SPU-IMSAF, $N=4, P=4, B=4, Q=3, \mu=0.08$ & $3.7 \times 10^{7}$ & 58.3 & 1900 \\
SM-SPU-IMSAF, $N=4, P=4, B=4, Q=3, \mu=0.34$ & $5.48 \times 10^{6}$ & 8.63 & 1700 \\
\hline
\end{tabular}

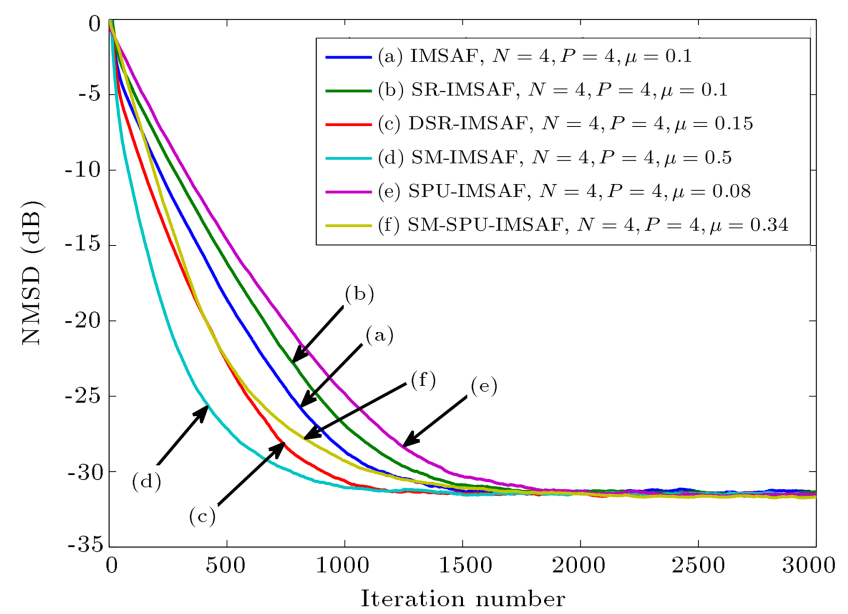

Figure 18. The Normalized Mean Square Deviation (NMSD) learning curves of Improved Multiband-Structured Subband Adaptive Filter (IMSAF), SR-IMSAF, DSR-IMSAF, proposed SPU-IMSAF, SM-IMSAF, and SM-SPU-IMSAF algorithms for the same steady-state error $(M=256, N=4, P=4, B=4$, $Q=3)$.

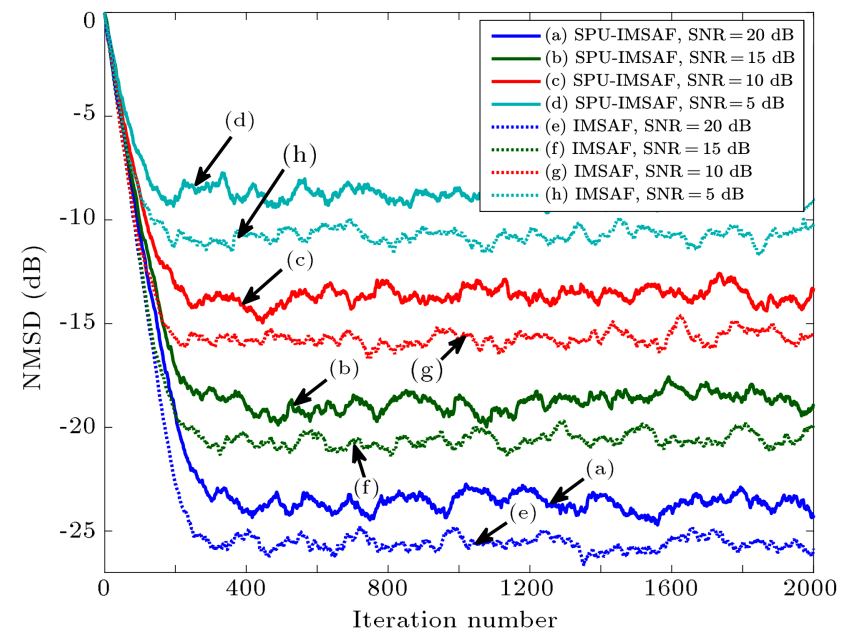

Figure 19. The Normalized Mean Square Deviation (NMSD) learning curves of Improved Multiband-Structured Subband Adaptive Filter (IMSAF) and SPU-IMSAF for different values of Signal to Noise Ratio (SNR).

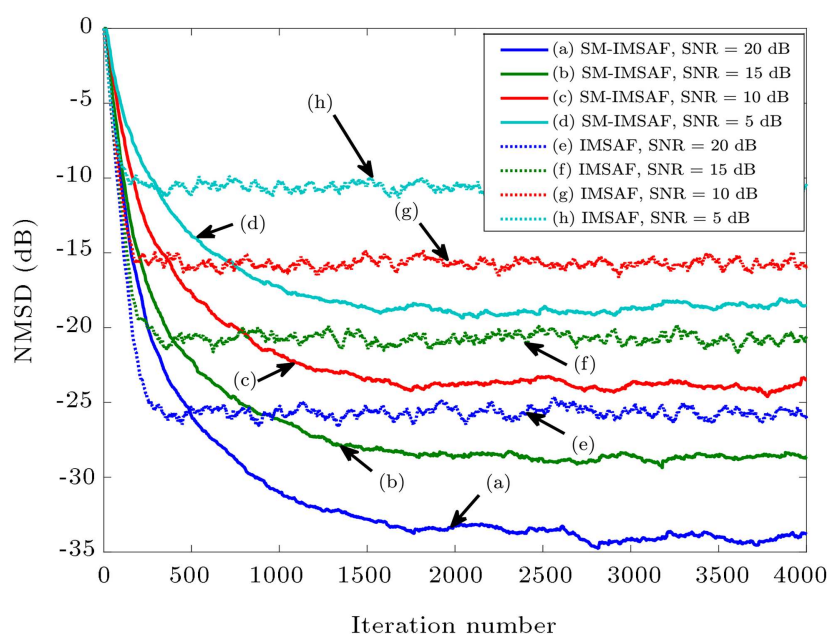

Figure 20. The Normalized Mean Square Deviation (NMSD) learning curves of Improved Multiband-Structured Subband Adaptive Filter (IMSAF) and SM-IMSAF for different values of Signal to Noise Ratio (SNR).

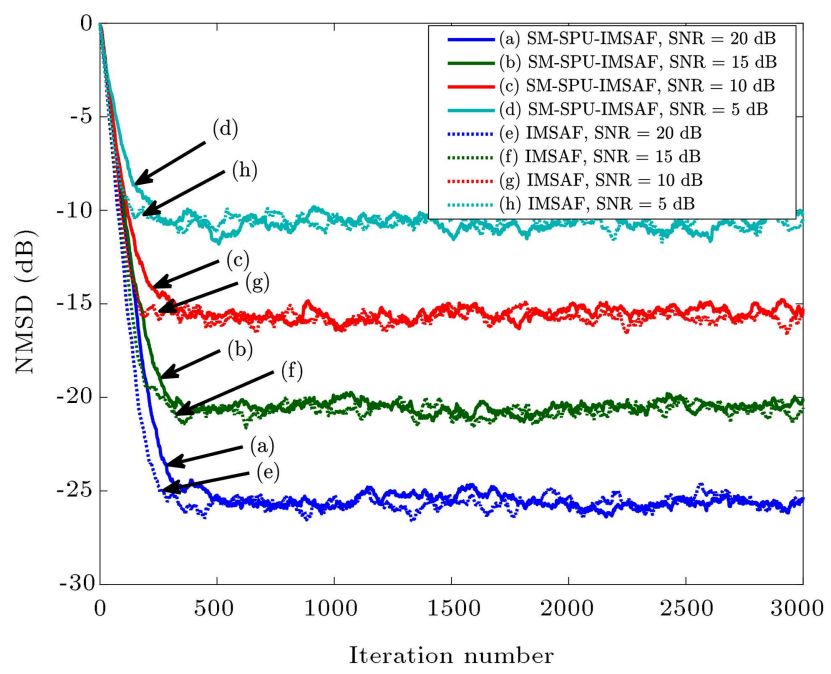

Figure 21. The Normalized Mean Square Deviation (NMSD) learning curves of Improved

Multiband-Structured Subband Adaptive Filter (IMSAF) and SM-SPU-IMSAF for different values of Signal to Noise Ratio (SNR). 


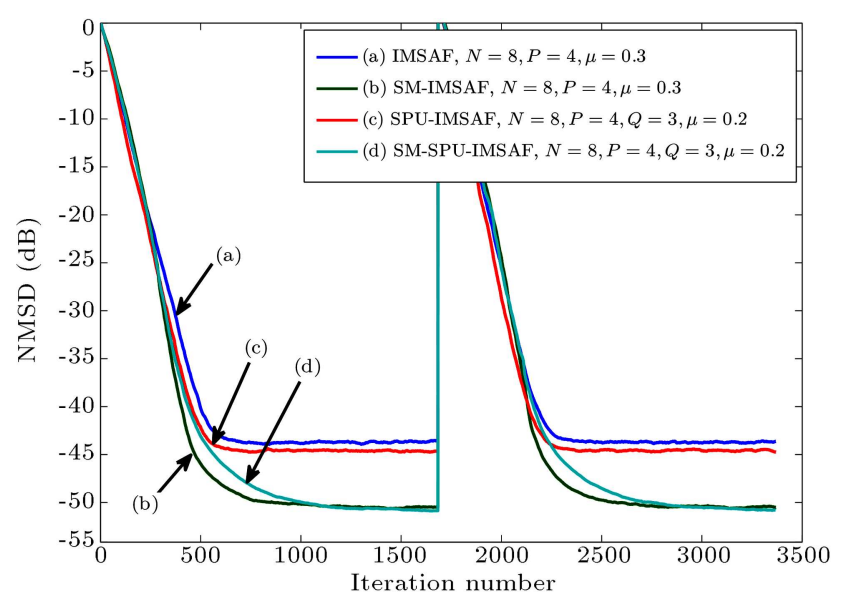

Figure 22. Tracking performance of the Improved Multiband-Structured Subband Adaptive Filter (IMSAF) and the proposed SPU-IMSAF, SM-IMSAF, and SM-SPU-IMSAF algorithms $(M=256, N=8, P=4$, $B=4, Q=3)$.

increases. The NMSD learning curves for IMSAF and SM-IMSAF are shown in Figure 20. Figure 21 shows the results for IMSAF and SM-SPU-IMSAF. The same performance given in Figure 19 can be seen for both figures. Figure 22 presents the tracking performances of IMSAF, SPU-IMSAF, SM-IMSAF, and SM-SPUIMSAF. For tracking performance analysis, we consider a system to identify the two unknown filters with $M=256$, whose $z$-domain transfer functions are given as follows:

$$
\mathbf{W}_{1}(z)=\sum_{n=0}^{127} z^{-n}-\sum_{n=128}^{M-1} z^{-n}
$$

and:

$$
\mathbf{W}_{2}(z)=-\sum_{n=0}^{M-1} z^{-n}
$$

where the transfer function of optimum filter coefficients will be $\mathbf{W}_{1}(z)$ for $n \leq 1700$, and the transfer function of optimum filter coefficients will be $\mathbf{W}_{2}(z)$ for $1700<n \leq 3400$. The parameters $N$ and $P$ were set to 8 and 4 , respectively. The NMSD learning curves show that the SM-IMSAF and SM-SPU-IMSAF algorithms have better tracking performance than IMSAF. Also, the SPU-IMSAF has closer performance to conventional IMSAF. Figure 23 presents the results for real speech input signal. The parameters $N$ and $P$ were set to 8 and the step-size was set to 0.5. Again, the SM-IMSAF and SM-SPU-IMSAF have a faster

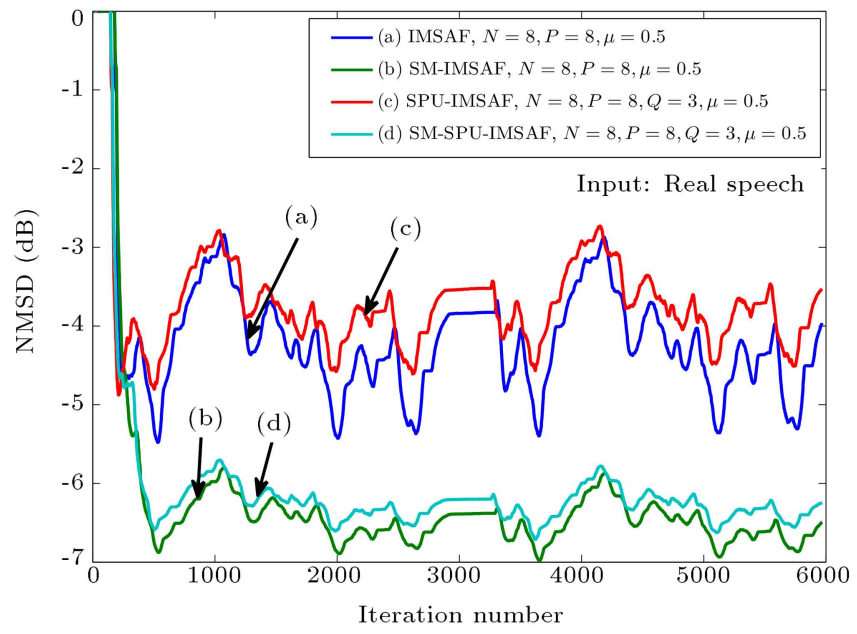

Figure 23. Normalized Mean Square Deviation (NMSD) learning curves of the Improved Multiband-Structured Subband Adaptive Filter (IMSAF) and the proposed SPU-IMSAF, SM-IMSAF, and SM-SPU-IMSAF algorithms for real speech input signal $(M=256, N=8$, $P=8, B=4, Q=3)$.

convergence rate than IMSAF. According to Figures 24 and 25, the adaptation is performed in SM-IMSAF and SM-SPU-IMSAF algorithms. These figures have been presented in the limited range of iterations for all subbands. Therefore, the computational complexity of SM-IMSAF and SM-SPU-IMSAF is lower than that of IMSAF. Again, in the worst case, the computational complexity of SM-IMSAF is the same as that of IMSAF. However, the performance of SM-IMSAF is better than conventional IMSAF algorithm.

Figures 26 and 27 evaluate the stability bounds of the proposed algorithms. Figure 26 shows the steadystate NMSD versus the step-size for SPU-IMSAF algorithm with $B=4$. Different values of $Q$ have been selected. At low values of $Q$, the stability bounds are low. Upon increasing the value of parameter $Q$, the stability bounds increase. In Figure 27, the steady-state NMSD values versus the step-size for IMSAF, SPUIMSAF, SM-IMSAF, and SM-SPU-IMSAF algorithms are presented. As is clear, the SM-IMSAF has a higher stability bound than the other algorithms.

Table 5 shows the maximum values for the stepsize $\left(\mu_{\max }\right)$ to guarantee the stability of the algorithms.

\section{Conclusion}

This paper proposed two new adaptive filter algorithms

Table 5. Stability bounds of Improved Multiband-Structured Subband Adaptive Filter (IMSAF), SPU-IMSAF, SM-SPU-IMSAF, and SM-IMSAF algorithms.

\begin{tabular}{ccccccc}
\hline Step-size & IMSAF & $\begin{array}{c}\text { SPU-IMSAF, } \\
\boldsymbol{Q}=\mathbf{1}\end{array}$ & $\begin{array}{c}\text { SPU-IMSAF, } \\
\boldsymbol{Q}=\mathbf{2}\end{array}$ & $\begin{array}{c}\text { SPU-IMSAF, } \\
\boldsymbol{Q}=\mathbf{3}\end{array}$ & $\begin{array}{c}\text { SM-SPU-IMSAF, } \\
\boldsymbol{Q}=\mathbf{3}\end{array}$ & SM-IMSAF \\
\hline$\mu_{\max }$ & 0.873 & 0.12 & 0.39 & 0.857 & 0.868 & 1.14 \\
\hline
\end{tabular}



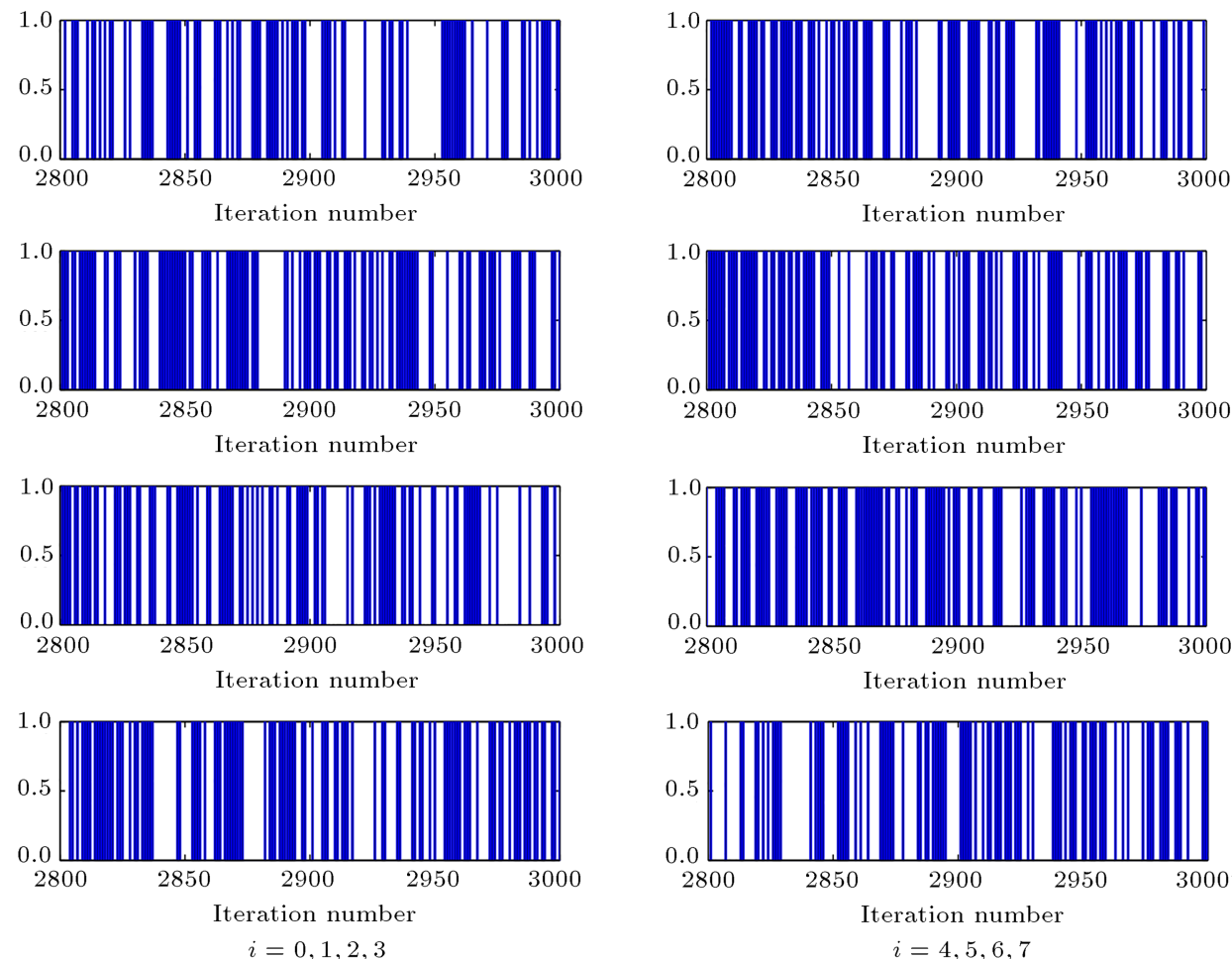

Figure 24. Filter coefficients in update for SM-IMSAF in different subbands $(M=256, P=8, N=8)$.
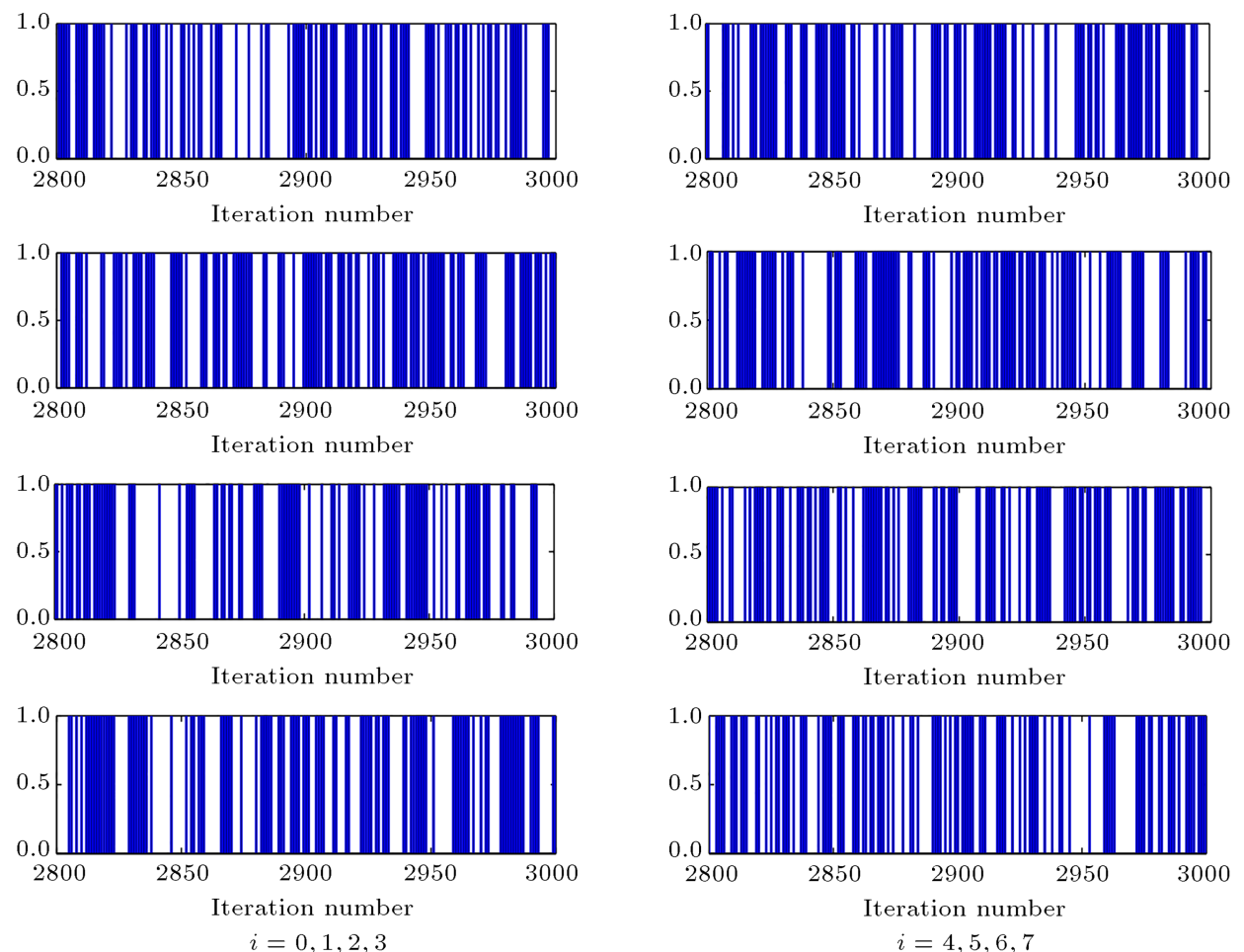

Figure 25. Filter coefficients in update for SM-SPU-IMSAF in different subbands $(M=256, P=8, N=8)$.

with low computational complexity feature. These algorithms utilized the Selective Partial Update (SPU) and Set-Membership (SM) approaches in Improved Multiband-Structured Subband Adaptive Filter (IM$\mathrm{SAF}$ ) algorithm. In SPU-IMSAF, a subset of filter coefficients was optimally selected and updated at each subband for every iteration. The SM-IMSAF had fast convergence speed, low steady-state error, and low computational complexity features at the same time. Also, by combining SM and SPU approaches, the SM- 


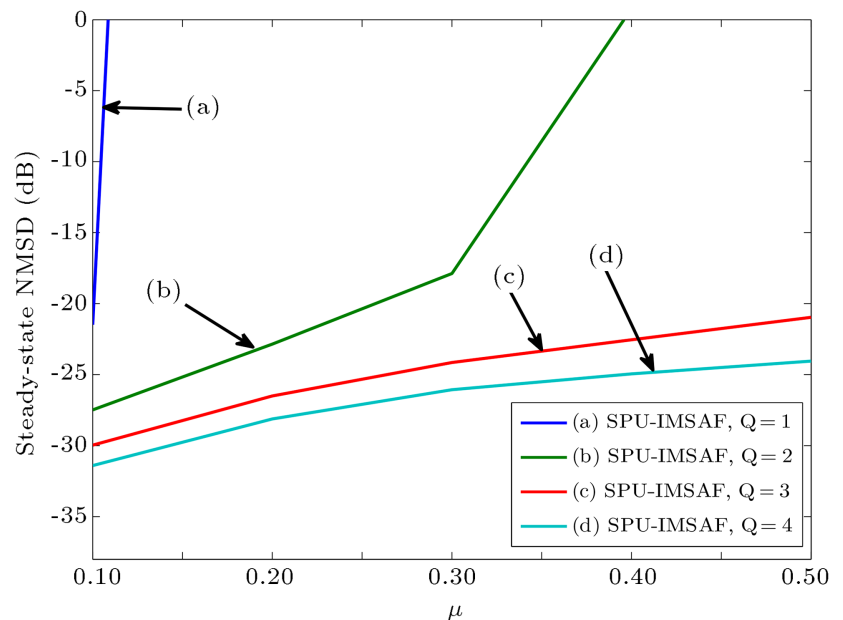

Figure 26. Steady-state Normalized Mean Square Deviation (NMSD) versus the step-size for SPU-IMSAF algorithm with different values of $Q$.

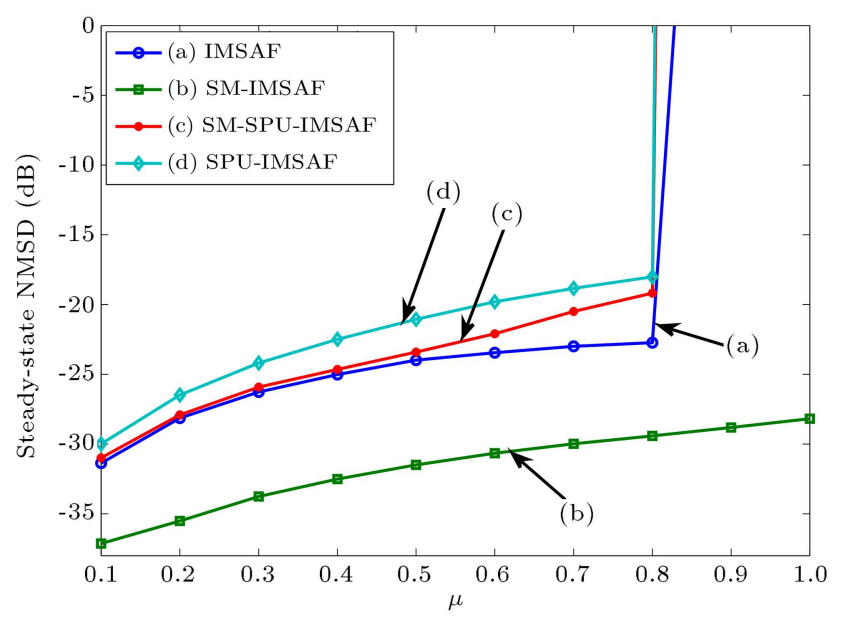

Figure 27. Steady-state Normalized Mean Square Deviation (NMSD) versus the step-size for Improved Multiband-Structured Subband Adaptive Filter (IMSAF), SM-IMSAF, SPU-IMSAF, and SM-SPU-IMSAF algorithms.

SPU-IMSAF was introduced. The good performance of the proposed algorithms was confirmed through several experiments.

\section{References}

1. Widrow, B. and Stearns, D., Adaptive Signal Processing, Englewood Cliffs, NJ Prentice Hall, Inc (1985).

2. Haykin, S.S., Adaptive Filter Theory, Pearson Education India, 5th Edn. (2013).

3. Sayed, A.H., Adaptive Filters, John Wiley \& Sons, Inc. (2008).

4. Farhang-Boroujeny, B., Adaptive Filters: Theory and Applications, John Wiley \& Sons, Ltd (2013).

5. Dogancay, K., Partial-Update Adaptive Signal Process- ing: Design Analysis and Implementation, Academic Press (2008).

6. Ozeki, K. and Umeda, T. "An adaptive filtering algorithm using an orthogonal projection to an affine subspace and its properties", Electron. Commun. Japan (Part I Commun.), 67, pp. 19-27 (1984).

7. Lee, K.-A. and Gan, W.-S. "Improving convergence of the NLMS algorithm using constrained subband updates", IEEE Signal Process. Lett., 11(9), pp. 736739 (2004).

8. Abadi, M.S.E. and Danaee, A.-R. "Low computational complexity family of affine projection algorithms over adaptive distributed incremental networks", $A E U$ International J. Electron. Commun., 68, pp. 97-110 (2014).

9. Shams Esfand Abadi, M. and Shafiee, M.S. "A family of diffusion normalized subband adaptive filter algorithms over distributed networks", Int. J. Commun. Syst., 30, pp. 1-15 (2017).

10. Lee, J.-W., Kim, S.-E., and Song, W.-J. "Dataselective diffusion LMS for reducing communication overhead", Signal Processing, 113, pp. 211-217 (2015).

11. Dogancay, K. and Tanrikulu, O. "Adaptive filtering algorithms with selective partial updates", IEEE Trans. Circuits Syst. II Analog Digit. Signal Process., 48(8), pp. $762-769$ (2001).

12. Aboulnasr, T. and Mayyas, K. "Complexity reduction of the NLMS algorithm via selective coefficient update", IEEE Trans. Signal Process., 47(5), pp. 14211424 (1999).

13. Werner, S., De Campos, M.L.R., and Diniz, P.S.R. "Partial-update NLMS algorithms with data-selective updating", IEEE Trans. Signal Process., 52(4), pp. 938-949 (2004).

14. Abadi, M.S.E., Mehrdad, V., and Gholipour, A. "A family of variable step-size affine projection adaptive filtering algorithms with selective regressors and selective partial updates", Sci. Iran. Trans. D, Comput. Sci. Eng. Electr., 17, pp. 81-98 (2010).

15. Arablouei, R., Werner, S., Huang, Y.-F., and Dogancay, K. "Distributed least mean-square estimation with partial diffusion", IEEE Trans. Signal Process., 62, pp. 472-484 (2013).

16. Abadi, M.S.E. and Husy, J.H. "Selective partial update and set-membership subband adaptive filters", Signal Processing, 88, pp. 2463-2471 (2008).

17. Desiraju, N.K., Doclo, S., and Wolff, T. "Efficient multichannel acoustic echo cancellation using constrained tap selection schemes in the subband domain", EURASIP J. Adv. Signal Process., 2017, p. 63 (2017).

18. Abadi, M.S.E., Shafiee, M.S., and Zalaghi, M. "A low computational complexity normalized subband adaptive filter algorithm employing signed regressor of input signal", EURASIP J. Adv. Signal Process., 2018(1), pp. 1-23 (2018). 
19. Cho, J., Baek, H.J., Park, B.Y., and Shin, J. "Variable step-size sign subband adaptive filter with subband filter selection", Signal Processing, 152, pp. 141-147 (2018).

20. Abadi, M.S.E. and Shafiee, M.S. "Diffusion normalized subband adaptive algorithm for distributed estimation employing signed regressor of input signal", Digit. Signal Process., 70, pp. 73-83 (2017).

21. Doust, H.A.M. and Shah, T.K. "NLMS algorithm with variable step-size using set-membership identification", Sci. Iran. Trans. D, Comput. Sci. Eng. Electr., 9, pp. 378-384 (2002).

22. Gollamudi, S., Nagaraj, S., Kapoor, S., and Huang, Y.-F. "Set-membership filtering and a set-membership normalized LMS algorithm with an adaptive step size", IEEE Signal Process. Lett., 5, pp. 111-114 (1998).

23. Werner, S. and Diniz, P.S.R. "Set-membership affine projection algorithm", IEEE Signal Process. Lett., 8, pp. 231-235 (2001).

24. Yang, F., Wu, M., Ji, P., and Yang, J. "An improved multiband-structured subband adaptive filter algorithm", IEEE Signal Process. Lett., 19(10), pp. 647-650 (2012).

25. Yang, F., Wu, M., Ji, P., and Yang, J. "Low- complexity implementation of the improved multibandstructured subband adaptive filter algorithm", IEEE Trans. Signal Process., 63(19), pp. 5133-5148 (2015).

26. Yang, F., Wu, M., Ji, P., Kuang, Z., and Yang, J. "Transient and steady-state analyses of the improved multiband-structured subband adaptive filter algorithm", IET Signal Process., 9, pp. 596-604 (2015).

27. Abadi, M.S.E., Husy, J.H., and Ahmadi, M.J. "Two improved multiband structured subband adaptive filter algorithms with reduced computational complexity", Signal Processing, 154, pp. 15-29 (2019).

28. Malvar, H.S., Signal Processing with Lapped Transforms, Artech House, Inc. (1992).

\section{Biographies}

Mohammad Shams Esfand Abadi received the BS degree in Electrical Engineering from Mazandaran University, Mazandaran, Iran and the MS degree in the same field of study from Tarbiat Modares University, Tehran, Iran in 2000 and 2002, respectively, and the $\mathrm{PhD}$ degree in biomedical engineering from Tarbiat Modares University in 2007. Since 2004, he has been with the Faculty of Electrical Engineering, Shahid Rajaee Teacher Training University, Tehran, Iran, where he is currently a Professor. His research interests include digital image processing, digital filter theory, adaptive distributed networks, and adaptive filter algorithms.

John Håkon Husøy was born in Toronto, ON, Canada in 1956. He received the MSc and $\mathrm{PhD}$ in Electrical Engineering in 1981 and 1991, respectively, from the Norwegian Institute of Technology, University of Trondheim, Trondheim, Norway. He has been involved in hardware and software development in various positions in several companies. Since 1992, he has been a Professor at the Department of Electrical and Computer Engineering, University of Stavanger, Norway. His research interests include adaptive algorithms, digital filtering, signal representations, image compression, bioelectrical signal processing, and image analysis.

Mohammad Javad Ahmadi was born in 1990. He received the BS degree (with honors) in Electrical Engineering from Babol University of Technology, Babol, Iran and the MSc degree in Communications from Sharif University of Technology, Tehran, Iran. His research interests include adaptive algorithms, estimation theory, and adaptive distributed networks. 